TRANSACTIONS OF THE

AMERICAN MATHEMATICAL SOCIETY

Volume 353, Number 3, Pages 1119-1149

S 0002-9947(00)02729-X

Article electronically published on November 14, 2000

\title{
TWO-WEIGHT NORM INEQUALITIES FOR CESÀRO MEANS OF LAGUERRE EXPANSIONS
}

\author{
BENJAMIN MUCKENHOUPT AND DAVID W. WEBB
}

\begin{abstract}
Two-weight $L^{p}$ norm inequalities are proved for Cesàro means of Laguerre polynomial series and for the supremum of these means. These extend known norm inequalities, even in the single power weight and "unweighted" cases, by including all values of $p \geq 1$ for all positive orders of the Cesàro summation and all values of the Laguerre parameter $\alpha>-1$. Almost everywhere convergence results are obtained as a corollary. For the Cesàro means the hypothesized conditions are shown to be necessary for the norm inequalities. Necessity results are also obtained for the norm inequalities with the supremum of the Cesàro means; in particular, for the single power weight case the conditions are necessary and sufficient for summation of order greater than one sixth.
\end{abstract}

\section{Introduction}

Let $\sigma_{n}^{(\alpha, \delta)}(f, x)$ be the $n$th Cesàro mean of order $\delta$ for the expansion of $f(x)$ in orthonormalized Laguerre polynomials $\mathcal{L}_{n}^{(\alpha)}(x)$. The purpose of this paper is to prove inequalities of the form

$$
\sup _{n \geq 0}\left\|\sigma_{n}^{(\alpha, \delta)}(f, x) x^{a}(1+x)^{b-a}\right\|_{p} \leq C\left\|f(x) x^{A}(1+x)^{B-A}\right\|_{p}
$$

for $\delta>0, \alpha>-1,1 \leq p \leq \infty$ and

$$
\left\|\sup _{n \geq 0}\left(\left|\sigma_{n}^{(\alpha, \delta)}(f, x)\right|\right) x^{a}(1+x)^{b-a}\right\|_{p} \leq C\left\|f(x) x^{A}(1+x)^{B-A}\right\|_{p}
$$

for $\delta>0, \alpha>-1,1<p \leq \infty$, where $\|g\|_{p}$ denotes unweighted $L^{p}$ norm of $g$ on $[0, \infty)$ and $C$ is independent of $f$. A weak type result for $\sup _{n \geq 0}\left|\sigma_{n}^{(\alpha, \delta)}(f, x)\right|$ is proved for $p=1$. Immediate consequences are mean convergence results and pointwise almost everywhere convergence results, and the latter are stronger than what can be obtained from (1.2) with the same weight on both sides. In their full generality the conditions on $a, b, A$ and $B$ required here to make (1.1) and (1.2) hold are complicated. Detailed statements can be found in $\S 2$. For many readers, however, the consequences of the general theorems that are stated in this section will be of more interest. These are the results with the single weight $x^{r}$ replacing the weights $x^{a}(1+x)^{b-a}$ and $x^{A}(1+x)^{B-A}$ in (1.1) and (1.2). Even these simple

Received by the editors May 28, 1999.

1991 Mathematics Subject Classification. Primary 42C10.

Key words and phrases. Cesàro means, Laguerre expansions, Laguerre polynomials, two-weight norm inequalities, weighted norm inequalities. 
consequences generalize and strengthen previous known results by allowing more values of $\alpha, \delta, p$ and $r$.

The norm inequalities proved here are based on a new estimate for the kernel of $\sigma_{n}^{(\alpha, \delta)}$ obtained in [14]. This has let us obtain conditions on $a, b, A$ and $B$ that are necessary and sufficient for (1.1) to hold. For (1.2) the conditions used are necessary and sufficient for the operator with the estimate as its kernel. For (1.2) thirteen inequalities are assumed in the sufficiency proof. Ten of these are shown to be necessary. One is not necessary, as shown in $\S 10$. For the other two we make no assertion concerning their necessity. However, slightly weaker versions of those two inequalities are proved necessary for (1.1), and, of course, these weaker versions are necessary for (1.2) since (1.2) implies (1.1).

Most authors have chosen the two weight functions in inequalities (1.1) and (1.2) to be the same and equal to various "natural" powers of $x$. Our results when the weight functions are a single power of $x$ are stated below as Theorems (1.3), (1.13), (1.16), (1.19) and (1.20). They are consequences of the more general Theorems (2.29), (2.30), (2.31), 2.34), (9.3) and (10.2); some comments on how this reduction is done in a few not entirely obvious cases are given at the end of $\S 2$. It is interesting to note that for the theorems stated here $x^{1 / 2-1 / p}$ is the most natural weight to use since for this weight all the restrictions on $r$ are satisfied for all $\alpha>-1$ and $\delta>0$. As a result, for this weight function and $\alpha>-1$, (1.4) holds for $4 /(6 \delta+3) \leq p \leq 4 /(1-6 \delta)$ if $0<\delta \leq 1 / 6$, 1.14) holds for $2 /(1+6 \delta)<p<2 /(1-6 \delta)$ if $0<\delta<1 / 6$ and for $1<p \leq \infty$ if $\delta \geq 1 / 6$. Furthermore, $\lim _{n \rightarrow \infty} \sigma_{n}^{(\alpha, \delta)}(f, x)=f(x)$ almost everywhere if $\alpha>-1, \delta>0$ and $\int_{0}^{\infty} x^{p / 2-1}|f(x)|^{p} d x<\infty$ for some $p$ satisfying $1 \leq p<\infty$.

Theorem (1.3). If $\alpha>-1,1 \leq p \leq \infty$ and $\delta>0$, then

$$
\sup _{n \geq 0}\left\|\sigma_{n}^{(\alpha, \delta)}(f, x) x^{r}\right\|_{p} \leq C\left\|f(x) x^{r}\right\|_{p}
$$

holds with $C$ independent of $f$ if and only if

$$
\begin{array}{lll}
\delta & \geq \frac{2}{3 p}-\frac{1}{2}, & \\
\delta & \geq \frac{1}{6}-\frac{2}{3 p}, & \\
r & >-\frac{1}{p}-\frac{\alpha}{2} & (\geq \text { if } p=\infty), \\
r & >\frac{1}{4}-\frac{\delta}{2}-\frac{1}{p}, & \left(>\text { if } \delta=\frac{2}{3 p}-\frac{1}{2}\right), \\
r & \geq-\frac{\delta}{2}-\frac{2}{3 p} & (\leq \text { if } p=1), \\
r & <1+\frac{\alpha}{2}-\frac{1}{p} \\
r & <\frac{3}{4}+\frac{\delta}{2}-\frac{1}{p} &
\end{array}
$$

and

$$
r \leq \frac{2}{3}+\frac{\delta}{2}-\frac{2}{3 p} \quad\left(<\text { if } \delta=\frac{1}{6}-\frac{2}{3 p}\right)
$$


Theorem (1.13). If $\alpha>-1,1<p \leq \infty$ and $\delta>1 / 6$, then

$$
\left\|\sup _{n \geq 0}\left(\left|\sigma_{n}^{(\alpha, \delta)}(f, x)\right|\right) x^{r}\right\|_{p} \leq C\left\|f(x) x^{r}\right\|_{p}
$$

holds with $C$ independent of $f$ if and only if (1.7)-(1.10) hold and

$$
r \leq \frac{2}{3}+\frac{\delta}{2}-\frac{1}{p} .
$$

These conditions are also sufficient for $\delta=1 / 6$ with the strengthened assumption that the inequality is strict in (1.15), and necessary for $0<\delta \leq 1 / 6$.

Theorem (1.16). If $0<\delta<1 / 6, \alpha>-1,2 /(1+6 \delta)<p<2 /(1-6 \delta)$,

$$
\frac{1}{2}-\frac{1}{p}+\max (-3 \delta(\alpha+1),-2 \delta)<r<\frac{1}{2}-\frac{1}{p}+\min \left(3 \delta(\alpha+1), \frac{3 \delta}{2}\right)
$$

and

$$
r>\max \left(\frac{1}{3}-\frac{2}{3 p}-\frac{5 \delta}{2}, \frac{1}{6}-\frac{1}{3 p}-\frac{7 \delta}{2}\right),
$$

then (1.14) holds with $C$ independent of $f$.

Theorem (1.19). If $\alpha>-1, \delta \geq 1 / 6,-1-\alpha / 2<r<\alpha / 2,-\delta / 2-2 / 3 \leq r \leq$ $\delta / 2-1 / 3$ with strict inequality in the last two if $\delta=1 / 6$ and $E(\mu)$ is the set where $x^{r} \sup _{n \geq 0}\left(\left|\sigma_{n}^{(\alpha, \delta)}(f, x)\right|\right)>\mu$, then $\left|E_{\mu}\right| \leq(C / \mu) \int_{0}^{\infty}|f(x)| x^{r} d x$ with $C$ independent of $f$ and $\mu$.

Theorems (1.13) and (1.19) imply almost everywhere convergence results. However, better results can be obtained from the two weight results. This is because almost everywhere convergence will follow from weak or strong type inequalities for $\sup _{n>0}\left(\left|\sigma_{n}^{(\alpha, \delta)}(f, x)\right|\right)$ for any weight on the left side of the type considered. Theorem (2.34) gives the following.

Theorem (1.20). If $\delta>0, \alpha>-1,1 \leq p<\infty$, $\left\|x^{r} f(x)\right\|_{p}<\infty, r \leq 3 / 4+$ $\delta / 2-1 / p$, (1.10), $r \geq-1 / 4-\delta-1 / 3 p(>$ if $p=4 / 3$ ) and $r \geq 1 / 4-\delta-1 / p$, then $\lim _{n \rightarrow \infty} \sigma_{n}^{(\alpha, \delta)}(f, x)=f(x)$ for almost every $x$.

Previous results concerning the inequalities (1.1) and (1.2) and almost everywhere convergence include the following. Poiani in Theorem 1 , page 10 of [7] considered the case $a=A$ and $b=B$ in (1.1) for $\delta=1$ and $\alpha>-1$. She obtained the same results as Theorem (2.29) when specialized to this case with some minor differences. She did not include the possibilities of $a=-\alpha / 2$ and $a=5 / 4$ when $p=\infty$ nor $a=\alpha / 2$ and $a=-5 / 4$ when $p=1$. In addition, she included the case $a+b=-4 /(3 p)+7 / 3, b=19 / 12-1 /(3 p)$ for $4<p \leq \infty$ and the case $a+b=-4 /(3 p)-1, b=-5 / 4-1 /(3 p)$ for $1 \leq p<4 / 3$; these cases violate the necessity conditions in Theorem (2.29).

Other authors have used a fixed power of $x$ as a weight function. Markett, Theorem 1, page 420 of [3] proved (1.4) for $r=0$ with $\alpha \geq 0$ and $0<\delta \leq 1 / 2$ provided

$$
\frac{1-2 \delta}{4}<\frac{1}{p}<\frac{3+2 \delta}{4}
$$


Theorem (1.3) gives this result for $\alpha>-1$ provided

$$
\max \left(\frac{1-2 \delta}{4}, \frac{-\alpha}{2}\right)<\frac{1}{p}<\min \left(\frac{3+2 \delta}{4}, \frac{\alpha+2}{2}\right) .
$$

Stempak in Theorem 1.1, page 318 of [10] obtained almost everywhere convergence of $\sigma_{n}^{(\alpha, \delta)}(f, x)$ for $\alpha \geq 0$ and $\delta>\alpha+2 / 3$ provided $\|f(x)\|_{p}<\infty$ and $1 \leq p<\infty$. Theorem (1.20) gives this almost everywhere convergence for $1 \leq p<\infty$ provided $\delta \geq \max (2 / p-3 / 2,1 / 4-1 / p), \alpha>-1$ and $\alpha>(2 / p)-2(\geq$ for $p=1)$.

Results with weight $x^{r}$ for $r=\alpha / p-\alpha / 2$ arise naturally if the functions $x^{-\alpha} \mathcal{L}_{n}^{(\alpha)}\left(x^{2}\right)$ are viewed as an orthogonal system on $[0, \infty)$ with measure $x^{2 \alpha+1} d x$ or the functions $x^{-\alpha / 2} \mathcal{L}_{n}^{(\alpha)}(x)$ are viewed as orthogonal with measure $x^{\alpha} d x$. Thangavelu in Theorem 6.2 .2 on page 145 of [12] considered an inequality equivalent to (1.4) with this value of $r$ for $\alpha \geq 0$. His result is that (1.4) holds for $1 \leq p \leq \infty$ if $\delta>\alpha+1 / 2$, and for $0<\delta \leq \alpha+1 / 2$ it holds provided

$$
\frac{4 \alpha+4}{2 \alpha+3+2 \delta}<p<\frac{4 \alpha+4}{2 \alpha+1-2 \delta}
$$

Theorem (1.3) gives this for $\alpha \geq 0$ and gives an extension to $-1<\alpha<0$. For $-1 / 3 \leq \alpha<0$ Theorem (1.3) gives the range of $p$ in (1.23) if $-\alpha /(4+6 \alpha) \leq \delta \leq$ $\alpha+1 / 2$ and

$$
\frac{4}{6 \delta+3} \leq p \leq \frac{4}{1-6 \delta}
$$

if $0<\delta<-\alpha /(4+6 \alpha)$. For $-1<\alpha<-1 / 3$ the result holds for $0<\delta \leq 1 / 6$ provided $p$ satisfies (1.24). (Note that the condition $(\alpha+2 / 3) / p>(\alpha-\delta) / 2$ resulting from (1.9) is implied by $(\alpha+1) / p>(1+2 \alpha-2 \delta) / 4$ for $\alpha \geq-2 / 3$ and by $1 / p<(1+6 \delta) / 2$ for $-1<\alpha<-2 / 3$. Similarly, the condition resulting from (1.12) is implied by the other conditions.)

Stempak studied (1.14) for this value of $r$. In the comments before Proposition 4.3, page 325 of [10] he obtained this for $\alpha \geq 0, \delta>0$ and $1 \leq p \leq \infty$ provided $p<\frac{4 \alpha+2}{1+2 \alpha-2 \delta}$ for $0<\delta<\alpha+1 / 2$ and $p>\frac{6 \alpha+4}{2+3 \alpha+3 \delta}$ for $0<\delta<\alpha+2 / 3$. Theorem (1.13) holds for this $r$ and $1 \leq p \leq \infty$ provided $p<\frac{4 \alpha+4}{1+2 \alpha-2 \delta}$ for $1 / 6 \leq \delta<\alpha+1 / 2$ and $p \geq \frac{6 \alpha+6}{4+3 \alpha+3 \delta}$ ( $>$ for $\delta=\frac{1}{6}$ ) for $1 / 6 \leq \delta<\alpha+2 / 3$. For $0<\delta<1 / 6$, Theorem (1.16) gives the ranges $\frac{2 \alpha+2}{\alpha+1+3 \delta}<p<\frac{2 \alpha+2}{\alpha+1-4 \delta}$ for $\alpha \geq-1 / 3, \frac{2 \alpha+2}{\alpha+1+3 \delta}<p<\frac{2}{1-6 \delta}$ for $-1 / 2 \leq \alpha \leq-1 / 3$ and $\frac{2}{1+6 \delta}<p<\frac{2}{1-6 \delta}$ for $-1<\alpha \leq-1 / 2$.

For this value of $r$, Stempak in Proposition 4.3, page 325 of [10] also proved that if $\left\|x^{r} f(x)\right\|_{p}<\infty$ then $\sigma_{n}^{(\alpha, \delta)}(f, x)$ converges to $f(x)$ for almost every $x$ provided $\alpha \geq 0, \delta>0,1 \leq p<\infty, p<\frac{4 \alpha+2}{1+2 \alpha-2 \delta}$ if $0<\delta<\alpha+1 / 2$ and $p>\frac{6 \alpha+4}{3 \alpha+3 \delta+2}$ if $0<\delta<\alpha+2 / 3$. For this value of $r$, Theorem (1.20) gives almost everywhere convergence for $\alpha>-1 / 2$ for the wider range

$$
\frac{4 \alpha+4}{3+2 \alpha+2 \delta} \leq p \leq \frac{4 \alpha+4}{1+2 \alpha-4 \delta}
$$

if $0<\delta<\alpha / 2+1 / 4, \frac{4 \alpha+4}{3+2 \alpha+2 \delta} \leq p<\infty$ if $\alpha / 2+1 / 4 \leq \delta<\alpha+1 / 2$ and $1 \leq p<\infty$ if $\delta \geq \alpha+1 / 2$. For $-1<\alpha \leq-1 / 2$ the convergence holds for $1 \leq p<\infty$.

The weight $x^{r}$ with $r=1 / 4-1 /(2 p)$ arises if the functions $(2 x)^{1 / 2} \mathcal{L}_{n}^{(\alpha)}\left(x^{2}\right)$ are taken as an orthonormal system on $[0, \infty)$ with Lebesgue measure. For this weight Markett, Theorem 2, page 22 of [4] proved (1.4) with $\alpha= \pm 1 / 2$ for $1 \leq p \leq \infty$ if $\delta>1 / 2$ and for $4 /(2 \delta+3)<p<4 /(1-2 \delta)$ if $0<\delta<1 / 2$. Thangavelu in Theorem 
1 , page 305 of [13] proved (1.4) with $\alpha \geq 1 / 2$ for $1 \leq p \leq \infty$ if $\delta>1 / 6$. Theorem (1.3) gives this result for $1 \leq p \leq \infty$ if $\alpha>-1 / 2$ and $\delta>1 / 6$; for $\alpha \leq-1 / 2$ it also requires $2 /(3+2 \alpha)<p<-2 /(1+2 \alpha)$ and for $\delta<1 / 6$ it also requires $4 /(3+6 \delta) \leq p \leq 4 /(1-6 \delta)$.

Almost everywhere convergence results for this weight include Theorem 1.2, page 318 of [10], which gives this convergence for $1 \leq p<\infty$ if $\left\|f(x) x^{r}\right\|_{p}<\infty, \alpha \geq 0$ and $\delta>\alpha+2 / 3$. Thangavelu in Theorem 3, page 306 of [13] proved this for $1 \leq p<\infty$ if $\alpha \geq 1 / 2$ and $\delta>1 / 6$. Theorem (1.20) gives this for $1 \leq p<\infty$ if $\delta>0$ and $\alpha \geq-1 / 2$; for $-1<\alpha<-1 / 2$ there is the additional condition $p>2 /(3+2 \alpha)$.

The sufficiency proofs of (1.1) and (1.2) given here are direct and based on the kernel estimate derived in [14. The complexity of this kernel estimate, however, requires the estimation of thirty-eight integral expressions. The resulting sufficient conditions are complicated but in the case of (1.1) they are in fact also necessary conditions. For (1.2) all but three of the fourteen inequalities used in the sufficiency proof are shown to be necessary. Those three are necessary conditions for the norm inequalilty for the operator based on the estimate. As shown in $\S 10$, one is not necessary for (1.2) to hold. We conjecture that the other two are also not necessary but note that they differ only slightly from conditions that are necessary.

The sufficiency proofs are given here as a series of lemmas in $\S \S 3-6$. For these proofs six basic lemmas suffice to obtain estimates of 28 of the 38 expressions that arise when estimating (1.2) and 24 of the 38 expressions that arise when estimating (1.1). These lemmas are proved for $p=1$ and $p=\infty$ in $\S 3$ and applied in $\S 4$ along with an interpolation argument. When the variables are close to $4 n+2 \alpha+2$ or to each other, the interpolation approach does not work and individual proofs are given for these parts. A look at the conditions shows why there are problems with an interpolation approach to all the parts. Some of the needed inequalities change at $p=4 / 3$ or $p=4$, and weak inequalities become strong at those values of $p$. An interpolation argument would require additional results for these two values of $p$, and the weak type results needed for such an interpolation are false.

Throughout this paper $C$ will be used for positive constants independent of $f$, $n, x$ and $y$ but not necessarily the same at every occurrence. Frequent use will be made, without further comment, of the fact that for $0<2 a<b$ and $r \neq-1$ we have $\int_{a}^{b} x^{r} d x \approx\left(a^{r+1}+b^{r+1}\right)$, where $\approx$ means that the symbol on the left is bounded above and below by $C$ times the expression on the right. Since all integrands are nonnegative, integration sets may be enlarged during estimation without mention. The symbol $p^{\prime}=p /(p-1)$, and $\chi_{E}(x)$ denotes the characteristic function of the set $E$.

\section{Definitions And Results for $1<p<\infty$}

Let the Laguerre polynomials $\left\{L_{n}^{(\alpha)}(x)\right\}$ be defined for $\alpha>-1$ and nonnegative integer $n$ by the orthogonality condition

$$
\int_{0}^{\infty} e^{-x} x^{\alpha} L_{n}^{(\alpha)}(x) L_{m}^{(\alpha)}(x) d x=\frac{\Gamma(n+\alpha+1)}{n !} \delta_{m n}
$$

and the requirement that the leading term of $L_{n}^{(\alpha)}(x)$ be $(-x)^{n} / n$ !. The orthonormal functions $\mathcal{L}_{n}^{(\alpha)}(x)$ are defined by 


$$
\mathcal{L}_{n}^{(\alpha)}(x)=\left(\frac{n !}{\Gamma(n+\alpha+1)}\right)^{1 / 2} e^{-x / 2} x^{\alpha / 2} L_{n}^{(\alpha)}(x) .
$$

For a fixed $\alpha>-1$, nonnegative integer $n$ and positive constants $\lambda, \eta$ and $\xi$ define $\nu=\nu(n)=4 n+2 \alpha+2$,

$$
\Phi_{n}^{(\alpha)}(x)=\left\{\begin{array}{lc}
1 & 0 \leq x \leq \nu \\
\exp \left(\frac{-\eta|\nu-x|^{3 / 2}}{\nu^{1 / 2}}\right) & \nu \leq x \leq(1+\lambda) \nu \\
e^{-\xi x} & (1+\lambda) \nu \leq x
\end{array}\right.
$$

and

$$
\mathcal{M}_{n}^{(\alpha)}(x)=x^{\alpha / 2}\left(\nu^{-1}+x\right)^{-1 / 4-\alpha / 2}\left(\nu^{1 / 3}+|x-\nu|\right)^{-1 / 4} \Phi_{n}^{(\alpha)}(x) .
$$

It is known, see 1] page 699 and the theorem on page 289 of [5], that given $\alpha>-1$ and $\lambda>0$, there exist positive constants $C, \eta$ and $\xi$ such that $\left|\mathcal{L}_{n}^{(\alpha)}(x)\right| \leq C \mathcal{M}_{n}^{(\alpha)}(x)$ holds for $x>0$ and $n \geq 0$ with $C$ independent of $x$ and $n$.

The $n$th Cesàro kernel of order $\delta$ is defined by

$$
\mathcal{K}_{n}^{(\alpha, \delta)}(x, y)=\frac{1}{A_{n}^{\delta}} \sum_{k=0}^{n} A_{n-k}^{\delta} \mathcal{L}_{k}^{(\alpha)}(x) \mathcal{L}_{k}^{(\alpha)}(y),
$$

where $A_{n}^{\delta}=\left(\begin{array}{c}n+\delta \\ n\end{array}\right)$ and

$$
\sigma_{n}^{(\alpha, \delta)}(f, x)=\int_{0}^{\infty} \mathcal{K}_{n}^{(\alpha, \delta)}(x, y) f(y) d y .
$$

To prove inequalities of the forms (1.1) and (1.2) we will use the fact from Theorem $\mathrm{C}$ in $\S 1$ of 14 that given $\alpha>-1$ and $\lambda>0$, there are positive numbers $\eta, \xi$ and $C$ such that for $n \geq 0, x>0$ and $y>0$

$$
\left|\mathcal{K}_{n}^{(\alpha, \delta)}(x, y)\right| \leq C G_{n}^{(\alpha, \delta)}(x, y)
$$

where

$$
\begin{aligned}
G_{n}^{(\alpha, \delta)}(x, y)= & \nu^{-\delta} \mathcal{M}_{n}^{(\alpha)}(x) \mathcal{M}_{n}^{(\alpha)}(y) \\
& \times\left(\frac{(x+y)\left(\nu^{1 / 3}+|x-\nu|+|y-\nu|\right)^{2}}{x+y+(x-y)^{2}\left(\nu^{1 / 3}+|x-\nu|+|y-\nu|\right)}\right)^{(1+\delta) / 2} .
\end{aligned}
$$

Upper bounds for the left sides of (1.1) and (1.2) can, therefore, be obtained by replacing $\mathcal{K}_{n}^{(\alpha, \delta)}(x, y)$ by $G_{n}^{(\alpha, \delta)}(x, y)$ and $f(y)$ by $|f(y)|$. It will be convenient to define $\tau=\tau(\alpha, \delta, \nu, x, y, a, b, A, B)$ by

$$
\tau=x^{a}(1+x)^{b-a} y^{-A}(1+y)^{A-B} G_{n}^{(\alpha, \delta)}(x, y) .
$$

To prove (1.1) it is sufficient to show that

$$
\sup _{n \geq 0}\left\|\int_{0}^{\infty} \tau|f(y)| d y\right\|_{p} \leq C\|f(x)\|_{p},
$$

and to prove (1.2) it is sufficient to show that

$$
\left\|\sup _{n \geq 0} \int_{0}^{\infty} \tau|f(y)| d y\right\|_{p} \leq C\|f(x)\|_{p} .
$$


To do this we will need various estimates of $\tau$. To make this easier for the reader we note that

$$
\begin{gathered}
\tau \approx \frac{\nu^{-\delta} x^{a+\alpha / 2}(1+x)^{b-a} y^{-A+\alpha / 2}(1+y)^{A-B} \Phi_{n}^{(\alpha)}(x) \Phi_{n}^{(\alpha)}(y)}{\left(\nu^{-1}+x\right)^{1 / 4+\alpha / 2}\left(\nu^{1 / 3}+|x-\nu|\right)^{1 / 4}\left(\nu^{-1}+y\right)^{1 / 4+\alpha / 2}\left(\nu^{1 / 3}+|y-\nu|\right)^{1 / 4}} \\
\times\left(\frac{(x+y)\left(\nu^{1 / 3}+|x-\nu|+|y-\nu|\right)^{2}}{x+y+(x-y)^{2}\left(\nu^{1 / 3}+|x-\nu|+|y-\nu|\right)}\right)^{(1+\delta) / 2} .
\end{gathered}
$$

The estimate to be used for $\Phi_{n}^{(\alpha)}$ in upper bounds for $\tau$ will be a simplified version with $\lambda=1 / 2$; we will estimate it as 1 when the argument is less than $3 \nu / 2$.

The sets of conditions that must be satisfied for the various theorems are fairly complex. We will, therefore, assign names to these conditions here as follows: $N_{p}$ for those used in estimates of the norm and $S_{p}$ for those used in estimates of the sup of the norm.

Parameters $(a, b, A, B, \alpha, \delta)$ will be said to satisfy the $N_{p}$ conditions provided

$$
\begin{aligned}
& a \geq \frac{1}{4}-\frac{\delta}{2}-\frac{1}{p}, \\
& a>-\frac{\alpha}{2}-\frac{1}{p} \quad(\geq \text { if } p=\infty), \\
& A-a \leq 0 \text {, } \\
& A \leq \frac{3}{4}+\frac{\delta}{2}-\frac{1}{p}, \\
& A<1+\frac{\alpha}{2}-\frac{1}{p} \quad(\leq \text { if } p=1), \\
& a+B \geq-\delta-\frac{4}{3 p}, \\
& a+B \geq \frac{1}{2}-\delta-\frac{2}{p}, \\
& A+b \leq \frac{3}{2}+\delta-\frac{2}{p}, \\
& A+b \leq \frac{4}{3}+\delta-\frac{4}{3 p}, \\
& b \leq \frac{3}{4}+\delta-\frac{1}{p}, \\
& b \leq \frac{7}{12}+\delta-\frac{1}{3 p}, \\
& b-B \leq \delta+\frac{1}{2}-\frac{2}{3 p}, \\
& b-B \leq 0, \\
& b-B \leq \delta-\frac{1}{6}+\frac{2}{3 p}, \\
& B \geq-\frac{1}{4}-\delta-\frac{1}{3 p}, \\
& B \geq \frac{1}{4}-\delta-\frac{1}{p}
\end{aligned}
$$

and in at least one of each of the following pairs the inequality is strict: (2.9) and 
(2.11) except for $p=1,(2.9)$ and (2.15), (2.11) and (2.12) except for $p=\infty,(2.12)$ and (2.16), (2.14) and (2.15), (2.14) and (2.23), (2.15) and (2.24) except for $p=\infty$, (2.16) and (2.17), (2.16) and (2.18) except for $p=1,(2.17)$ and (2.19), (2.18) and (2.19), (2.19) and (2.22), (2.20) and (2.23), (2.23) and (2.24).

Parameters $(a, b, A, B, \alpha, \delta)$ will be said to satisfy the $S_{p}$ conditions provided they satisfy inequalities (2.10)-(2.15), 2.21), 2.23)-2.24),

$$
\begin{aligned}
a & >\frac{1}{4}-\frac{\delta}{2}-\frac{1}{p} \quad(\geq \text { if } p=\infty), \\
A+b & \leq \frac{4}{3}+\delta-\frac{2}{p}, \\
b & <\frac{7}{12}+\delta-\frac{1}{p} \quad(\leq \text { if } p=\infty), \\
b-B & \leq \delta-\frac{1}{6},
\end{aligned}
$$

and in at least one of each of the following pairs the inequality is strict: (2.11) and (2.12) except for $p=\infty$, (2.11) and (2.13), (2.11) and (2.25), (2.13) and (2.26), (2.14) and (2.15), (2.14) and (2.23), (2.15) and (2.24) except for $p=\infty,(2.15)$ and (2.25), (2.23) and (2.24), (2.23) and (2.28) for $p=1$, (2.26) and (2.27), (2.27) and $(2.28)$.

The main results are the following.

Theorem (2.29). If $1 \leq p \leq \infty, \alpha>-1$ and $\delta>0$, then (1.1) holds with $C$ independent of $f$ if and only if $(a, b, A, B, \alpha, \delta)$ satisfy the $N_{p}$ conditions.

Theorem (2.30). If $1<p \leq \infty, \alpha>-1, \delta>0$ and $(a, b, A, B, \alpha, \delta)$ satisfy the $S_{p}$ conditions, then (1.2) holds with $C$ independent of $f$.

As a substitute for Theorem (2.30) when $p=1$ we have the following.

Theorem (2.31). If $\alpha>-1, \delta>0,(a, b, A, B, \alpha, \delta)$ satisfy the $S_{1}$ conditions and $E_{\mu}$ is the set where $x^{a}(1+x)^{b-a} \sup _{n \geq 0}\left(\left|\sigma_{n}^{(\alpha, \delta)}(f, x)\right|\right)>\mu$, then $\left|E_{\mu}\right| \leq$ $(C / \mu)\left\|f(x) x^{A}(1+x)^{B-A}\right\|_{1}$ holds with $C$ independent of $f$ and $\mu$.

Because of (2.3) and (2.5), Theorem (2.30) is an immediate consequence of the following result.

Theorem (2.32). If $1<p \leq \infty, \alpha>-1$ and $\delta>0$, then

$$
\left\|\sup _{\nu \geq 2+2 \alpha} \int_{0}^{\infty} \tau|f(y)| d y\right\|_{p} \leq C\|f(x)\|_{p}
$$

(with $C$ independent of $f$ ) if and only if $(a, b, A, B, \alpha, \delta)$ satisfy the $S_{p}$ conditions.

The following is an immediate consequence of Theorems (2.30) and (2.31) since with the hypotheses of Theorem (2.34) parameters $a$ and $b$ can be chosen to satisfy the $S_{p}$ conditions, and Lebesgue measure is absolutely continuous with respect to $x^{a}(1+x)^{b-a} d x$ for any $a$ and $b$.

Theorem (2.34). If $1 \leq p<\infty, \alpha>-1, \delta>0$, (2.12), (2.13), 2.23) and (2.24) are satisfied with equality in at most one of (2.23) and (2.24) and

$$
\left\|f(x) x^{A}(1+x)^{B}\right\|_{p}<\infty
$$

then $\lim _{n \rightarrow \infty} \sigma_{n}^{(\alpha, \delta)}(f, x)=f(x)$ for almost every $x>0$. 
Sufficiency in Theorem (2.29) will be obtained by proving (2.6); this is enough because of (2.3) and (2.5). To avoid technical problems, the sufficiency parts of Theorem (2.29) and (2.32) will be proved with the sup's taken over $\nu \geq 4$. This can be done because for $2+2 \alpha \leq \nu \leq 4$ the inequality

$$
\tau(\alpha, \delta, \nu, x, y, a, b, A, B) \leq C \tau(\alpha, \delta, 4, x, y, a, b, A, B)
$$

holds with $C$ independent of $x$ and $y$. The proof will be done by majorizing $\tau$ by a sum of parts and proving the sufficiency for each part. The parts will be denoted as $\tau_{j}$ for $1 \leq j \leq 12$. Each part will be taken equal to $\tau$ if certain conditions are met, and 0 otherwise. The conditions for each part are given below. It is easy to verify that for a given $\nu \geq 4$ every pair $(x, y)$ of positive real numbers satisfies at least one of the conditions.

Part Number

Conditions

$\begin{array}{rcc}1 & 0 \leq x \leq 2 / \nu & 0 \leq y \leq 2 / \nu \\ 2 & 2 / \nu \leq x \leq 1 & 0 \leq y \leq x / 2 \\ 3 & 1 \leq x \leq \nu / 2 & 0 \leq y \leq x / 2 \\ 4 & 3 \nu / 2 \leq x & 0 \leq y \leq x / 2 \\ 5 & 2 / \nu \leq y \leq 1 & 0 \leq x \leq y / 2 \\ 6 & 1 \leq y \leq \nu / 2 & 0 \leq x \leq y / 2 \\ 7 & 3 \nu / 2 \leq y & 0 \leq x \leq y / 2 \\ 8 & \nu / 2 \leq x \leq 3 \nu / 2 & 0 \leq y \leq x / 2 \\ 9 & \nu / 2 \leq y \leq 3 \nu / 2 & 0 \leq x \leq y / 2 \\ 10 & x / 2 \leq y \leq 2 x & 3 / \nu \leq x+y \leq \nu \\ 11 & x / 2 \leq y \leq 2 x & \nu \leq x+y \leq 3 \nu \\ 12 & x / 2 \leq y \leq 2 x & 3 \nu \leq x+y\end{array}$

Sufficiency in Theorem (2.29) will be proved by showing with its hypotheses that

$$
\sup _{\nu \geq 4}\left\|\int_{0}^{\infty} \tau_{j}|f(y)| d y\right\|_{p} \leq C\|f(x)\|_{p}
$$

holds for $1 \leq j \leq 12$. This is done in $\S \S 4-6$. For Theorem (2.31) and the sufficiency portion of Theorem (2.32) we also show in $\S \S 4-6$ if $1 \leq p \leq \infty$ that

$$
\left\|\sup _{\nu \geq 4} \int_{0}^{\infty} \tau_{j}|f(y)| d y\right\|_{p} \leq C\|f(x)\|_{p}
$$

for $1 \leq j \leq 9$ and that

$$
\int_{0}^{\infty} \tau_{j}|f(y)| d y \leq C M f(x)
$$

for $10 \leq j \leq 12$, where $M f(x)$ denotes the Hardy-Littlewood maximal function of $f$ at $x$ and $C$ is independent of $\nu, x$ and $f$. These and standard facts about the maximal function, Theorem 1, page 5 of [8], then complete the proof of Theorem (2.31) and the sufficiency in Theorem (2.32). Proofs of the necessity results in Theorems (2.29) and (2.32) are given in $\S \S 7-9$. The additional fact that (2.26) is a necessary condition for (1.2), Theorem (9.3), is also proved in $\S 9$. Finally, in 
$\S 10$ we show that (2.28) is not a necessary condition for (1.2) and prove (1.2) with hypotheses that do not include (2.28) .

The theorems in $\S 1$ are straightforward specializations of the theorems of this section and Theorem (9.3) but a few comments may help the reader through the details. In Theorem (1.3), for example, the condition $r \leq 7 / 12+\delta-1 /(3 p)$ resulting from (2.19) would seem to be needed as an additional hypothesis. It is not needed because the conditions (1.12) and (1.6) imply it. Similarly, (1.9) and (1.5) imply $r \geq-1 / 4-\delta-1 /(3 p)$ which comes from (2.23). The fact that strict inequality is needed in (1.8) follows from the fact that (2.9) and (2.15) can not both be equalities. Similarly the need for strict inequality in (1.11) follows from the fact that (2.12) and (2.16) can not both be equalities. The inequality (1.9) and the requirement of strict inequality if $\delta=2 /(3 p)-1 / 2$ follow from (2.14) and the condition that 2.14) and (2.23) can not both be equalities. Similarly, (2.17) and the pair condition between it and (2.19) produce (1.12). Considerations of the same sort prove Theorems (1.13) and (1.19).

\section{BASIC LEMMAS}

Of the 38 parts that will be estimated to prove the sufficiency portion of Theorems (2.29) and (2.32), a majority can be reduced to an interpolation argument based on six lemmas. For convenient reference, these lemmas are stated together here with the proofs given after the last one. It may be of interest to note that in the last four the conditions given are necessary and sufficient. This is also the case for lemmas (3.1) and (3.2) except for the fact that they are also true with suitable conditions for $s=0$. Since in the applications of those lemmas $s=-\delta / 2$, which is always strictly negative, there was no reason to add the complication of the case $s=0$. Note that even for these simple lemmas the peculiar conditions appear that in certain pairs of inequalities at least one must be strict.

Lemma (3.1). If $s<0, r+s<-1$ and $r+s+t \leq-1$, then for $p=1$

$$
\left\|x^{r} \chi_{[1, \infty)}(x) \sup _{\nu \geq x} \nu^{s} \int_{1}^{x} y^{t}|f(y)| d y\right\|_{p} \leq C\|f(x)\|_{p}
$$

with $C$ independent of $f$. If $s<0, r+s \leq 0$ and $r+s+t \leq-1$, with equality holding in at most one of the last two inequalities, this holds for $p=\infty$. In addition, if $s<0, r+s=-1$ and $r+s+t \leq-1$, then

$$
\sup _{\nu \geq 1} \nu^{s}\left\|x^{r} \chi_{[1, \nu]}(x) \int_{1}^{x} y^{t}|f(y)| d y\right\|_{1} \leq C\|f(x)\|_{1}
$$

with $C$ independent of $f$.

Lemma (3.2). If $s<0, s+t \leq 0$ and $r+s+t \leq-1$ with equality holding in at most one of the last two inequalities, then for $p=1$

$$
\left\|x^{r} \chi_{[1, \infty)}(x) \sup _{\nu \geq x} \nu^{s} \int_{x}^{\nu} y^{t}|f(y)| d y\right\|_{p} \leq C\|f(x)\|_{p}
$$

with $C$ independent of $f$. If $s<0, s+t \leq-1$ and $r+s+t \leq-1$, this holds for $p=\infty$. 
Lemma (3.3). If $t \leq 0, s+t \leq 0$ and $r+s+t \leq-1$ with strict inequalilty holding in the first two in case the third is an equality, then for $p=1$

$$
\left\|x^{r} \chi_{[1, \infty)}(x) \sup _{\nu \geq x} \nu^{s} \int_{\nu}^{\infty} y^{t}|f(y)| d y\right\|_{p} \leq C\|f(x)\|_{p}
$$

with $C$ independent of $f$. If $t<-1, s+t \leq-1$ and $r+s+t \leq-1$, then this holds for $p=\infty$. In addition, if $t=0, s+t<0$ and $r+s+t=-1$, then

$$
\sup _{\nu \geq 1} \nu^{s}\left\|x^{r} \chi_{[1, \nu]}(x) \int_{\nu}^{\infty} y^{t}|f(y)| d y\right\|_{1} \leq C\|f(x)\|_{1} .
$$

Lemma (3.4). If $r<-1, r+s<-1$ and $r+s+t \leq-1$, then for $p=1$

$$
\left\|x^{r} \chi_{[1, \infty)}(x) \sup _{1 \leq \nu \leq x} \nu^{s} \int_{1}^{\nu} y^{t}|f(y)| d y\right\|_{p} \leq C\|f(x)\|_{p}
$$

with $C$ independent of $f$. If $r \leq 0, r+s \leq 0$ and $r+s+t \leq-1$ with equality in at most one of the last two inequalities, then this holds for $p=\infty$. In addition, if $r<-1, r+s=-1$ and $r+s+t \leq-1$, then

$$
\sup _{\nu \geq 1} \nu^{s}\left\|x^{r} \chi_{[\nu, \infty)}(x) \int_{1}^{\nu} y^{t}|f(y)| d y\right\|_{1} \leq C\|f(x)\|_{1}
$$

with $C$ independent of $f$.

Lemma (3.5). If $r<-1, r+t \leq-1$ and $r+s+t \leq-1$, then for $p=1$

$$
\left\|x^{r} \chi_{[1, \infty)}(x) \sup _{1 \leq \nu \leq x} \nu^{s} \int_{\nu}^{x} y^{t}|f(y)| d y\right\|_{p} \leq C\|f(x)\|_{p}
$$

with $C$ independent of $f$. If $r \leq 0, r+t \leq-1$ and $r+s+t \leq-1$ with equality holding in at most one of the first two inequalities, then this holds for $p=\infty$.

Lemma (3.6). If $t \leq 0, r+t \leq-1$ and $r+s+t \leq-1$, with strict inequality in the last two in case of equality in the first, then for $p=1$

$$
\left\|x^{r} \chi_{[1, \infty)}(x) \sup _{1 \leq \nu \leq x} \nu^{s} \int_{x}^{\infty} y^{t}|f(y)| d y\right\|_{p} \leq C\|f(x)\|_{p}
$$

with $C$ independent of $f$. If $t<-1, r+t \leq-1$ and $r+s+t \leq-1$, then this holds for $p=\infty$. In addition, if $t=0, r+t<-1$ and $r+s+t=-1$, then

$$
\sup _{\nu \geq 1} \nu^{s}\left\|x^{r} \chi_{[\nu, \infty]}(x) \int_{x}^{\infty} y^{t}|f(y)| d y\right\|_{1} \leq C\|f(x)\|_{1}
$$

with $C$ independent of $f$.

The proofs of the assertions in these lemmas for $p=\infty$ are all done by replacing $|f(y)|$ on the left by $\|f(y)\|_{\infty}$ and factoring it out. The proofs are then completed by considering separately the cases $t=-1$ and $t \neq-1$ in the simple evaluations; this is left to the reader.

The first part of Lemma (3.1) for $p=1$ is proved by replacing $\nu^{s}$ by $x^{s}$ and reversing the order of integration. For the second part, replace $y^{t}$ by $1+\nu^{t}$ and the upper limit on the inner integral by $\infty$. The proof is completed by performing the $x$ integration; note that the conditions imply $r>-1$. 
To prove Lemma (B.2) for $p=1$, replace $\nu^{s}$ by $y^{s}$ inside the inner integral to get the bound

$$
C \int_{1}^{\infty} x^{r} \int_{x}^{\infty} y^{s+t}|f(y)| d y d x
$$

If $r+s+t=-1$, then since by hypothesis $s+t<0$ it follows that $r>-1$. Interchanging the order of integration leads immediately to the result. If $r+s+$ $t<-1$, replace $y^{s+t}$ by $x^{s+t}$ in the inner integral and interchange the order of integration to get the result.

For the first part of Lemma (3.3) with $p=1$, if $r+s+t<-1$, replace $y^{t}$ by $\nu^{t}$, the resulting $\nu^{s+t}$ by $x^{s+t}$ and the lower limit $\nu$ on the inner integral by 1 . Performing the $x$ integration then completes this case. If $r+s+t=-1$, then replace $\nu^{s}$ by $x^{s}$ if $s \leq 0$ and by $y^{s}$ in the inner integral if $s>0$. Next replace the lower limit in the inner integral by $x$. The conditions imply that the resulting exponents of $x, r$ or $r+s$ are greater than -1 , and interchanging the order of integration completes the proof. For the second part the hypotheses imply $r>-1$, and performing the outer integration leads immediately to the result since $r+s+1=-t=0$.

The first part of Lemma (3.4) with $p=1$ is proved by replacing $\nu^{s}$ by $x^{s}$ if $s \geq 0$ and by $y^{s}$ in the inner integral if $s<0$. Next change the upper limit of the inner integral to $x$ and interchange the order of integration to complete the proof. For the second part evaluate the outer integral and note that the resulting exponent of $\nu$ is 0 and that $t \leq 0$ to complete this part.

Lemma (3.5) for $p=1$ follows by replacing $\nu^{s}$ by $1+y^{s}$ in the inner integral, changing the lower limit of integration from $\nu$ to 1 and reversing the order of integration.

For the first part of Lemma (3.6) with $p=1$ first replace $\nu^{s}$ by $x^{\max (0, s)}$ and drop the sup. If $r+\max (s, 0)=-1$, then by hypothesis $t<0$ and $r+t+\max (s, 0)<$ -1 . Replacing $y^{t}$ by $x^{t}$, changing the lower limit of the inner integral to 1 and performing the outer integration will complete the proof. If $r+\max (s, 0) \neq-1$, then interchange the order of integration to get the result. For the second part change the lower limit of the inner integral to 1 and perform the outer integration to prove the result.

\section{Parts Reducible to the basic lemmas}

This section contains the proofs of the inequality (2.36) with the $N_{p}$ conditions for $1 \leq j \leq 7$ and of (2.37) with the $S_{p}$ conditions for $1 \leq j \leq 8$. This will be done by using interpolation between $p=1$ and $p=\infty$. For this the $N_{p}$ and $S_{p}$ conditions are not suitable. We will make use of the following weaker conditions.

Parameters $(a, b, A, B, \alpha, \delta)$ will be said to satisfy the $n_{p}$ conditions provided they satisfy the conditions (2.9) $-(2.13),(2.15)-(2.16),(2.18),(2.21),(2.24)$ and in at least one of each of the following pairs the inequality is strict: (2.9) and (2.11) except for $p=1$, (2.9) and (2.15), (2.11) and (2.12) except for $p=\infty,(2.12)$ and (2.16), (2.15) and (2.24) except for $p=\infty,(2.16)$ and (2.18) except for $p=1$.

Parameters $(a, b, A, B, \alpha, \delta)$ will be said to satisfy the $s_{p}$ conditions provided they satisfy the conditions (2.10)-(2.13), (2.15), (2.21), (2.24), (2.25)-(2.28) and in at least one of each of the following pairs the inequality is strict: (2.11) and (2.12) except for $p=\infty$, (2.11) and (2.13), (2.11) and (2.25), (2.13) and (2.26), (2.15) and (2.24) except for $p=\infty,(2.15)$ and (2.25), (2.26) and (2.27), (2.27) and (2.28).

In this section we will prove the following. 
Lemma (4.1). If $\alpha>-1, \delta>0,1 \leq j \leq 7$ and $(a, b, A, B, \alpha, \delta)$ satisfy the $n_{1}$ conditions, then (2.36) holds for $p=1$ with $C$ independent of $f$.

Lemma (4.2). If $\alpha>-1, \delta>0,1 \leq j \leq 8$ and $(a, b, A, B, \alpha, \delta)$ satisfy the $s_{1}$ conditions, then (2.37) holds for $p=1$ with $C$ independent of $f$.

Lemma (4.3). If $\alpha>-1, \delta>0,1 \leq j \leq 7$ and $(a, b, A, B, \alpha, \delta)$ satisfy the $n_{\infty}$ conditions, then (2.37) holds for $p=\infty$ with $C$ independent of $f$. This also holds for $j=8$ if the $s_{\infty}$ conditions are satisfied.

Corollary (4.4). If $1 \leq p \leq \infty, \alpha>-1, \delta>0,(a, b, A, B, \alpha, \delta)$ satisfy the $N_{p}$ conditions and $1 \leq j \leq 7$, then (2.36) holds with $C$ independent of $f$.

Corollary (4.5). If $1 \leq p \leq \infty, \alpha>-1, \delta>0,(a, b, A, B, \alpha, \delta)$ satisfy the $S_{p}$ conditions and $1 \leq j \leq 8$, then (2.37) holds with $C$ independent of $f$.

Corollary (4.4) follows by first observing that if $1<p<\infty$ and $(a, b, A, B, \alpha, \delta)$ satisfy the $N_{p}$ conditions, then $(a-1+1 / p, b-1+1 / p, A-1+1 / p, B-1+1 / p, \alpha, \delta)$ satisfy the $n_{1}$ conditions. Therefore, by Lemma (4.1) we have

$$
\int_{0}^{\infty} x^{a+1 / p}(1+x)^{b-a} \int_{0}^{\infty} \kappa_{j}|f(y)| d y \frac{d x}{x} \leq C \int_{0}^{\infty} x^{A+1 / p}(1+x)^{B-A}|f(x)| \frac{d x}{x},
$$

where $\kappa_{j}=G_{n}^{(\alpha, \delta)}(x, y)$, the unweighted kernel estimated defined in (2.4), on the set where $\tau_{j}>0$ and 0 elsewhere. Similarly, $(a+1 / p, b+1 / p, A+1 / p, B+1 / p, \alpha, \delta)$ satisfy the $n_{\infty}$ conditions. Therefore, we also have

$$
\left\|x^{a+1 / p}(1+x)^{b-a} \int_{0}^{\infty} \kappa_{j}|f(y)| d y\right\|_{\infty} \leq C\left\|x^{A+1 / p}(1+x)^{B-A} f(x)\right\|_{\infty} .
$$

Since $\kappa_{j}$ is independent of $a, b, A$ and $B$ the Marcinkiewicz interpolation theorem, Theorem 2.4, page 184 of [9], can be applied to get

$$
\begin{aligned}
& \int_{0}^{\infty}\left(x^{a+1 / p}\right.\left.(1+x)^{b-a} \int_{0}^{\infty} \kappa_{j}|f(y)| d y\right)^{p} \frac{d x}{x} \\
& \leq C \int_{0}^{\infty}\left(x^{A+1 / p}(1+x)^{B-A}|f(x)|\right)^{p} \frac{d x}{x}
\end{aligned}
$$

for $1<p<\infty$. This completes the proof of Corollary (4.4). Corollary 4.5 is proved in the same way from Lemmas (4.2) and (4.3) using the fact that if the $s_{\infty}$ conditions are satisfied, then the $n_{\infty}$ conditions are also satisfied.

Lemmas (4.1)-(4.3) will now be proved by considering each $j$ separately. For $1 \leq j \leq 7$ we will first show that (2.37) holds for $p=1$ if the $s_{1}$ conditions are satisfied and for $p=\infty$ if the $n_{\infty}$ conditions are satisfied. This will, also imply (2.36) under the same conditions. For parts where the $n_{1}$ conditions are less restrictive, the inequality (2.36) will then be proved for the additional cases allowed by the $n_{1}$ conditions. For $j=8$ only (2.37) will be proved using the $s_{1}$ and $s_{\infty}$ conditions.

For $j=1$, use of (2.8) shows that the left side of (2.37) is bounded by

$$
C\left\|x^{a+\alpha / 2} \chi_{[0,1 / 2]}(x) \sup _{4 \leq \nu \leq 2 / x} \nu^{\alpha+1} \int_{0}^{2 / \nu} y^{-A+\alpha / 2}|f(y)| d y\right\|_{p} .
$$

To estimate this, make the change of variables $x=2 / u$ and $y=2 / v$ to obtain

$$
C\left\|u^{-a-\frac{\alpha}{2}-\frac{2}{p}} \chi_{[4, \infty)}(u) \sup _{4 \leq \nu \leq u} \nu^{\alpha+1} \int_{\nu}^{\infty} v^{A-\frac{\alpha}{2}+\frac{2}{p}-2} g(v) d v\right\|_{p, u},
$$


where \|\|$_{p, u}$ denotes the $L^{p}$ norm in $u$, and

$$
g(v)=v^{-2 / p}\left|f\left(v^{-1}\right)\right| .
$$

The function $g(v)$ has the same $L^{p}$ norm as $f$. This notation will be used throughout this section. The inner integral should now be split at $u$. Lemma (3.5) can then be applied to the first part and Lemma 3.6 to the second part. The required conditions reduce to (2.10), (2.11), (2.13) and $A-a \leq 1+\alpha$ which is implied by (2.11) and is always strict because of the assumption that $\alpha>-1$. The only pair condition needed is that (2.11) and (2.13) can not both be equalities if $p=1$. This completes the proof of Lemmas (4.2) and (4.3) for $j=1$. For Lemma (4.1) with $j=1$ the same procedure and lemmas can be used; the pair condition on (2.11) and 2.13 is not needed.

For $j=2$ the left side of 2.37) is bounded by

$$
C\left\|x^{a-\frac{3}{4}-\frac{\delta}{2}} \chi_{[0,1]}(x) \sup _{\nu \geq 2 / x} \nu^{-\frac{\delta}{2}} \int_{0}^{x / 2} y^{-A+\frac{\alpha}{2}}\left(\nu^{-1}+y\right)^{-\frac{1}{4}-\frac{\alpha}{2}}|f(y)| d y\right\|_{p} .
$$

The change of variables $x=2 / u, y=2 / v$ gives the bound

$$
C\left\|u^{-a+\frac{3}{4}+\frac{\delta}{2}-\frac{2}{p}} \chi_{[2, \infty)}(u) \sup _{\nu \geq u} \nu^{-\frac{\delta}{2}} \int_{2 u}^{\infty} v^{A-\frac{\alpha}{2}+\frac{2}{p}-2}\left(\nu^{-1}+v^{-1}\right)^{-\frac{1}{4}-\frac{\alpha}{2}} g(v) d v\right\|_{p, u} .
$$

Now replace the lower limit in the inner integral by $u$ and split that integral at $\nu$. This gives as an estimate the sum of

$$
C\left\|u^{-a+\frac{3}{4}+\frac{\delta}{2}-\frac{2}{p}} \chi_{[2, \infty)}(u) \sup _{\nu \geq u} \nu^{-\frac{\delta}{2}} \int_{u}^{\nu} v^{A+\frac{2}{p}-\frac{7}{4}} g(v) d v\right\|_{p, u}
$$

and

$$
C\left\|u^{-a+\frac{3}{4}+\frac{\delta}{2}-\frac{2}{p}} \chi_{[2, \infty)}(u) \sup _{\nu \geq u} \nu^{\frac{1}{4}+\frac{\alpha}{2}-\frac{\delta}{2}} \int_{\nu}^{\infty} v^{A-\frac{\alpha}{2}+\frac{2}{p}-2} g(v) d v\right\|_{p, u} .
$$

Lemma (3.2) can be used on the first and Lemma 3.3 on the second. The required conditions for $p=1$ are $\delta>0$ which is an hypothesis, (2.11), 2.12) and (2.13), and that one of (2.11) and (2.12) and one of (2.11) and (2.13) must be strict. For $p=\infty$ the same inequalities are needed but the pair restrictions are not. This completes the proof of Lemmas (4.2) and (4.3) for $j=2$. Lemma (4.1) for $j=2$ follows in the same way; the requirement that one of (2.11) and (2.13) be strict is not needed.

For $j=3$ the left side of (2.37) is bounded by

$$
C\left\|x^{b-\frac{3}{4}-\frac{\delta}{2}} \chi_{[1, \infty)}(x) \sup _{\nu \geq 2 x} \nu^{-\frac{\delta}{2}} \int_{0}^{x / 2} y^{\frac{\alpha}{2}-A}\left(\nu^{-1}+y\right)^{-\frac{1}{4}-\frac{\alpha}{2}}(1+y)^{A-B}|f(y)| d y\right\|_{p} .
$$

To estimate this, change the upper limit in the inner integral to $x$ and split that integral at 1 . In the part from 0 to 1 make the change of variables $y=1 / v$. This shows that the left side of (2.37) is bounded by the sum of

$$
C\left\|x^{b-\frac{3}{4}-\frac{\delta}{2}} \chi_{[1, \infty]}(x) \sup _{\nu \geq 2 x} \nu^{-\frac{\delta}{2}} \int_{1}^{\infty} v^{A-\frac{\alpha}{2}-2+\frac{2}{p}}\left(\nu^{-1}+v^{-1}\right)^{-\frac{1}{4}-\frac{\alpha}{2}} g(v) d v\right\|_{p}
$$


and

$$
C\left\|x^{b-\frac{3}{4}-\frac{\delta}{2}} \chi_{[1, \infty]}(x) \sup _{\nu \geq 2 x} \nu^{-\frac{\delta}{2}} \int_{1}^{x} y^{-B-\frac{1}{4}}|f(y)| d y\right\|_{p} .
$$

Splitting the inner integral in (4.6) at $\nu$ shows that (4.6) is bounded by the sum of

$$
C\left\|x^{b-\frac{3}{4}-\frac{\delta}{2}} \chi_{[1, \infty]}(x) \sup _{\nu \geq 2 x} \nu^{-\frac{\delta}{2}} \int_{1}^{\nu} v^{A-\frac{7}{4}+\frac{2}{p}} g(v) d v\right\|_{p}
$$

and

$$
C\left\|x^{b-\frac{3}{4}-\frac{\delta}{2}} \chi_{[1, \infty]}(x) \sup _{\nu \geq 2 x} \nu^{\frac{\alpha}{2}+\frac{1}{4}-\frac{\delta}{2}} \int_{\nu}^{\infty} v^{A-\frac{\alpha}{2}-2+\frac{2}{p}} g(v) d v\right\|_{p} .
$$

Lemma (3.1) is used for (4.7). For $p=1$ we need $\delta>0, b<\delta-1 / 4$ and $b-B \leq \delta$; the last two follow from (2.27) and (2.21). For $p=\infty$ the inequalities $\delta>0, b \leq \delta+3 / 4$ and $b-B<\delta$ are sufficient, and the last two follow from (2.19) and (2.27). For (4.8) the inner integral will be split at $x$. For the first part Lemma (3.1) is used and requires for $p=1$ the first two conditions in the last part and $A+b \leq \delta-1 / 2$ which is implied by (2.26). For $p=\infty$ it requires $\delta>0,(2.18)$ and (2.16) with equality in at most one of the last two as hypothesized. For the second part of (4.8) with $p=1$ Lemma (3.2) requires $\delta>0$, (2.12) and $A+b \leq \delta-1 / 2$ with equality in at most one of the last two. Since (2.26) implies that the last condition holds strictly, these conditions are satisfied. For $p=\infty$ we need $\delta>0$, (2.12) and (2.16). Finally, Lemma (3.3) estimates (4.9). The requirements for $p=1$ are (2.13), (2.12) and $A+b \leq \delta-1 / 2$ with the last strict if either of the others is an equality. Since the hypothesized (2.26) implies the last is strict, this completes this part for $p=1$. For $p=\infty$ we need (2.13), (2.12) and (2.16) which are in the $n_{\infty}$ conditions. This completes the proof of Lemmas (4.2) and (4.3) for $j=3$.

The proof of Lemma (4.1) for $j=3$ uses the same breakup and the same lemmas. For this version of (4.7) the requirements are $\delta>0$, 2.18) and $b-B \leq \delta$. Since (2.21) has been assumed, the inequality $b-B<\delta$ holds. For the first part of (4.8) the requirements are $\delta>0,(2.16)$ and (2.18). For the second part of (4.8) the requirements are $\delta>0,(2.12)$ and (2.16) with equality in at most one of the last two as assumed in $n_{1}$. For (4.9) the requirements are (2.13), (2.12) and (2.16) with equality in at most one of the last two. These are also included in the $n_{1}$ conditions; this completes the proof of Lemma (4.1) for $j=3$.

The left side of (2.37) for $j=4$ has the bound

$$
C\left\|x^{b-\frac{1}{2}} e^{-\xi x} \chi_{[6, \infty)}(x) \sup _{4 \leq \nu \leq 2 x / 3} \nu^{-\delta-\frac{1}{12}} \int_{0}^{x / 2} \frac{y^{\frac{\alpha}{2}-A}(1+y)^{A-B}|f(y)| d y}{\left(\nu^{-1}+y\right)^{\frac{1}{4}+\frac{\alpha}{2}}}\right\|_{p} .
$$

The term $e^{-\xi x}$ can be replaced by $C x^{-q}$ with $q$ arbitrarily large. The same split in the inner integral and change of variables in the first part as done for the case $j=3$ can be done here. Lemmas (3.4), (3.5) and (3.6) can then be applied. Since the exponent of $x$ can be taken arbitrarily small, all the requirements of these lemmas except for the first inequality in Lemma (3.6) are automatically satisfied, and that requirement is just (2.13). It is simpler, however, to just apply Hölder's inequality to the inner integral to get the estimate. 
For $j=5$ the left side of (2.37) has the bound

$$
C\left\|x^{a+\frac{\alpha}{2}} \chi_{[0,1 / 2]}(x) \sup _{\nu \geq 4}\left(\nu^{-1}+x\right)^{-\frac{1}{4}-\frac{\alpha}{2}} \nu^{-\frac{\delta}{2}} \int_{\max (2 / \nu, 2 x)}^{1} y^{-A-\frac{3}{4}-\frac{\delta}{2}}|f(y)| d y\right\|_{p} .
$$

To estimate this, bound the sup by the sum of the sup's over $4 \leq \nu \leq 1 / x$ and $\nu \geq 1 / x$. Next make the changes of variables $x=1 / u$ and $y=1 / v$. This will give the sum of

$$
C\left\|u^{-a-\frac{\alpha}{2}-\frac{2}{p}} \chi_{[2, \infty)}(u) \sup _{2 \leq \nu \leq u} \nu^{\frac{1}{4}+\frac{\alpha}{2}-\frac{\delta}{2}} \int_{1}^{\nu / 2} v^{A-\frac{5}{4}+\frac{\delta}{2}+\frac{2}{p}} g(v) d v\right\|_{p, u}
$$

and

$$
C\left\|u^{\frac{1}{4}-\frac{2}{p}-a} \chi_{[2, \infty)}(u) \sup _{\nu \geq u} \nu^{-\frac{\delta}{2}} \int_{1}^{u / 2} v^{A-\frac{5}{4}+\frac{\delta}{2}+\frac{2}{p}} g(v) d v\right\|_{p, u}
$$

as an upper bound. For (4.10) with $p=1$ Lemma (3.4) requires (2.10), (2.25) and (2.11) for boundedness. For $p=\infty$ Lemma (3.4) requires (2.10), (2.9) and (2.11) and it also requires that one of (2.9) and (2.11) is strict. For (4.11) with $p=1$ Lemma (3.1) requires $\delta>0,(2.25)$ and (2.11). For $p=\infty$ it requires $\delta>0$, (2.9) and (2.11) and that one of the last two is strict. The estimation for the left side of (2.36) for $p=1$ and $j=5$ is similar. The outer integral is split at $1 / \nu$, and the same change of variables leads to versions of (4.10) and (4.11) with $\sup _{\nu \geq 4}$ outside the outer integral and the outer integrations respectively from $\nu$ to $\infty$ and from 1 to $\nu$. As before, Lemmas (3.4) and (3.1) are used. The requirements are $\delta>0$, (2.10), (2.9) and (2.11). This completes part 5 .

For $j=6$ the bound on the left side of (2.37) is

$$
\left\|\sup _{\nu \geq \max (4,4 x)} \frac{x^{a+\frac{\alpha}{2}}(1+x)^{b-a}}{\nu^{\frac{\delta}{2}}\left(\nu^{-1}+x\right)^{\frac{1}{4}+\frac{\alpha}{2}}} \chi_{[0, \infty]}(x) \int_{\max (2 x, 1)}^{\nu / 2} y^{-B-\frac{3}{4}-\frac{\delta}{2}}|f(y)| d y\right\|_{p} .
$$

Split the outer norm at 1 . In the first part make the change of variables $x=1 / u$ and split the sup into sup's over $4 \leq \nu \leq u$ and $\nu \geq u$. This gives an upper bound of the sum of

$$
\begin{gathered}
C\left\|u^{-a-\frac{\alpha}{2}-\frac{2}{p}} \chi_{[4, \infty)}(u) \sup _{4 \leq \nu \leq u} \nu^{\frac{1}{4}+\frac{\alpha}{2}-\frac{\delta}{2}} \int_{1}^{\nu} y^{-B-\frac{3}{4}-\frac{\delta}{2}}|f(y)| d y\right\|_{p, u}, \\
C\left\|u^{\frac{1}{4}-a-\frac{2}{p}} \chi_{[1, \infty)}(u) \sup _{\nu \geq u} \nu^{-\frac{\delta}{2}} \int_{1}^{\nu} y^{-B-\frac{3}{4}-\frac{\delta}{2}}|f(y)| d y\right\|_{p, u}
\end{gathered}
$$

and

$$
C\left\|x^{b-\frac{1}{4}} \chi_{[1, \infty)}(x) \sup _{\nu \geq x} \nu^{-\frac{\delta}{2}} \int_{x}^{\nu} y^{-B-\frac{3}{4}-\frac{\delta}{2}}|f(y)| d y\right\|_{p} .
$$

For (4.12) with $p=1$ Lemma (3.4) gives the bound with the assumption of (2.10), (2.25) and (2.15); with $p=\infty$ inequalities (2.10), (2.9) and (2.15) are needed plus the requirement that (2.9) and (2.15) are not both equalities. For (4.13) the inner integral should be split at $u$. Then Lemma (3.1) can be used on the first part and Lemma (3.2) on the second part. The requirements are $\delta>0,(2.25),(2.15)$ and (2.24) with equality in at most one of the last two for the case $p=1$. For $p=\infty$ the requirements are $\delta>0,(2.9),(2.15)$ and (2.24) with equality in at most one of (2.9) 
and (2.15). Lemma (3.2) estimates (4.14); for $p=1$ and $p=\infty$ the requirements are $\delta>0$, (2.24) and $b-B \leq \delta$ with equality in at most one of the last two for $p=1$. The last inequality and the equality condition follow from (2.21). The same approach proves Lemma (4.1) for $j=6$. The inequalities used are $\delta>0$, (2.9), (2.10), (2.15), (2.21) and (2.24) along with the requirement that equality can not occur in both (2.15) and (2.24).

For $j=7$ the left side of (2.36) has the bound

$$
C\left\|\sup _{\nu \geq 4} \frac{\nu^{-\delta-\frac{1}{12}} x^{a+\frac{\alpha}{2}}(1+x)^{b-a}}{\left(\nu^{-1}+x\right)^{\frac{1}{4}+\frac{\alpha}{2}}} \chi_{[0, \infty)}(x) \int_{\max (2 x, 3 \nu / 2)}^{\infty} y^{-B-\frac{1}{2}} e^{-\xi y}|f(y)| d y\right\|_{p} .
$$

The term $e^{-\xi y}$ can be replaced by $C y^{-q}$ with $q$ arbitrarily large. The same approach as used for $j=6$ can be used, and Lemmas (3.3), (3.5) and (3.6) will give the result with the only requirement being (2.10). As in the case $j=4$, however, it is easier to just use Hölder's inequality on the inner integral.

The left side of (2.37) with $j=8$ is bounded by

$$
C\left\|\chi_{[2, \infty)}(x) \sup _{2 x / 3 \leq \nu \leq 2 x} \frac{x^{b-\frac{1}{4}} \nu^{-\delta-\frac{1}{4}}}{\left(\nu^{\frac{1}{3}}+|x-\nu|\right)^{\frac{1}{4}}} \int_{0}^{x / 2} \frac{y^{\frac{\alpha}{2}-A}(1+y)^{A-B}}{\left(\nu^{-1}+y\right)^{\frac{1}{4}+\frac{\alpha}{2}}}|f(y)| d y\right\|_{p} .
$$

Now replace $\left(\nu^{\frac{1}{3}}+|x-\nu|\right)^{\frac{1}{4}}$ by $\nu^{\frac{1}{12}}$ and make the change of variables $u=2 x / 3$. This gives an upper bound of

$$
C\left\|\chi_{[1, \infty)}(u) \sup _{\nu \geq u} u^{b-\frac{1}{4}} \nu^{-\frac{1}{3}-\delta} \int_{0}^{u} y^{\frac{\alpha}{2}-A}\left(\nu^{-1}+y\right)^{-\frac{1}{4}-\frac{\alpha}{2}}(1+y)^{A-B}|f(y)| d y\right\|_{p, u} .
$$

The rest of the estimation is done in the way that the case $j=3$ was done. For $p=1$ the requirements are $\delta>-1 / 3,(2.13),(2.26)-(2.28)$,

$$
A \leq \delta+\frac{1}{12}
$$

$(2.26)$ and (4.15) are not both equalities and (2.13) and (2.26) are not both equalities. Since (2.12) has been assumed and implies that (4.15) holds strictly, this completes Lemma (4.2) for $j=8$. For $p=\infty$ the requirements are $\delta>-1 / 3$, (2.13), (2.26) -2.28), $A \leq \delta+13 / 12$ which is implied by (2.12), one of (2.26) and (2.27) must be strict and one of (2.27) and (2.28) must be strict. Since these conditions are contained in the $s_{\infty}$ conditions, this proves Lemma (4.3) for $j=8$.

\section{Parts With ONe Variable NeAR $\nu$}

This section contains the proof of the following lemma. A different approach is needed because the estimates contain powers of $|x-\nu|$ or $|y-\nu|$. Note that since the $S_{p}$ conditions imply the $N_{p}$ conditions, Lemma (5.1) also shows that the $S_{p}$ conditions imply (2.37) for $j=9$. Similarly, since (2.37) implies (2.36), this lemma also shows that the $N_{p}$ conditions imply (2.36).

Lemma (5.1). If $1 \leq p \leq \infty, \alpha>-1, \delta>0$ and $(a, b, A, B, \alpha, \delta)$ satisfy the $N_{p}$ conditions then (2.36) holds for $j=8$ and (2.37) holds for $j=9$. 
To prove (2.36) for $j=8$ we start with the fact that the left side has the bound

$$
C \sup _{\nu \geq 4}\left\|\frac{x^{b-\frac{1}{4}} \nu^{-\delta-\frac{1}{4}} \chi_{[\nu / 2,3 \nu / 2]}(x)}{\left(\nu^{1 / 3}+|x-\nu|\right)^{1 / 4}} \int_{0}^{x / 2} \frac{y^{\frac{\alpha}{2}-A}(1+y)^{A-B}|f(y)|}{\left(\nu^{-1}+y\right)^{\frac{1}{4}+\frac{\alpha}{2}}} d y\right\|_{p} .
$$

Now replace $x^{b-\frac{1}{4}}$ by $\nu$ to the same power and replace the upper limit of the inner integral by $\nu$. Then evaluate the $x$ integration and apply Hölder's inequality to the inner integral. Routine computation making use of (2.13) shows that if neither $A$ nor $B$ equals $3 / 4-1 / p$ and $p \neq 4$, then (5.2) has a bound of $\|f\|_{p}$ times

$$
C \sup _{\nu \geq 4} \nu^{b-\frac{1}{2}-\delta}\left(\nu^{\frac{1}{p}-\frac{1}{4}}+\nu^{\frac{1}{3 p}-\frac{1}{12}}\right)\left(1+\nu^{A-\frac{3}{4}+\frac{1}{p}}+\nu^{-B+\frac{3}{4}-\frac{1}{p}}\right) .
$$

If $A$ equals $3 / 4-1 / p$, the term $\nu^{A-\frac{3}{4}+\frac{1}{p}}$ is replaced by $(\log \nu)^{\frac{1}{p^{\prime}}}$. If $B$ equals $3 / 4-$ $1 / p$, the term $\nu^{-B+\frac{3}{4}-\frac{1}{p}}$ is replaced by $(\log \nu)^{\frac{1}{p^{\prime}}}$. If $p=4$, the term $\left(\nu^{\frac{1}{p}-\frac{1}{4}}+\nu^{\frac{1}{3 p}-\frac{1}{12}}\right)$ is replaced by $(\log \nu)^{\frac{1}{4}}$. If the terms in (15.3) are multiplied out, it follows from (2.16) -(2.19), (2.21) and (2.22) that all the resulting powers of $\nu$ are nonpositive. This completes the proof except for the special cases when there are log terms. If $A=3 / 4-1 / p$ and $p>1$, we need to show in addition that inequalities (2.18) and (2.19) are strict. The first follows from the fact that for this value of $A$ inequalities (2.16) and (2.18) are the same. By hypothesis equality can not occur in both if $p>1$; therefore, both must be strict. The second follows from the fact that (2.17) and (2.19) are the same and equality can not occur in both. Similarly, if $B=3 / 4-1 / p$, we must also have strict inequality in 2.18) and 2.19). The first follows from (2.21) and the fact that $\delta>0$. For this value of $B$ inequalities (2.19) and (2.22) are the same, and it follows from the hypothesis that these can not both be equalities that (2.19) is strict. Finally if $p=4$ we need to show that (2.16) and (2.18) are strict and $b-B<\delta$. For $p=4$ inequalities (2.16) and (2.17) are the same so both are strict. Similarly, (2.18) and (2.19) are the same and, therefore, strict. The inequality $b-B<\delta$ as mentioned before holds for all $B$ because of (2.21). This completes the proof of (2.36) for $j=8$.

For $j=9$ the left side of (2.37) has the bound

$$
C\left\|\sup _{\nu \geq 4} \chi_{[0,3 \nu / 4]}(x) \frac{x^{a+\frac{\alpha}{2}}(1+x)^{b-a}}{\left(\nu^{-1}+x\right)^{\frac{\alpha}{2}+\frac{1}{4}}} \int_{\nu / 2}^{3 \nu / 2} \frac{y^{-B-\frac{1}{2}-\delta}}{\left(\nu^{1 / 3}+|y-\nu|\right)^{1 / 4}}|f(y)| d y\right\|_{p} .
$$

Applying Hölder's inequality to the inner integral shows this has the bound

$$
C\left\|\sup _{\nu \geq 4} \chi_{[0, \nu]}(x) \frac{x^{a+\frac{\alpha}{2}}(1+x)^{b-a}}{\left(\nu^{-1}+x\right)^{\frac{\alpha}{2}+\frac{1}{4}}} H(\nu)\right\| \chi_{[\nu / 2,3 \nu / 2]} f(y)\left\|_{p, y}\right\|_{p, x},
$$

where \|\|$_{p, x}$ and \|\|$_{p, y}$ denote the unweighted $L^{p}$ norms with respect to $x$ and $y$ respectively and

$$
H(\nu)=\left\{\begin{array}{lr}
\nu^{-\frac{1}{4}-\delta-B-\frac{1}{3 p}} & 1 \leq p<4 / 3 \\
\nu^{-\frac{1}{2}-\delta-B}(\log \nu)^{\frac{1}{4}} & p=4 / 3 \\
\nu^{\frac{1}{4}-\delta-B-\frac{1}{p}} & 4 / 3<p \leq \infty .
\end{array}\right.
$$

To estimate (5.4) move the terms between the sup and the inner norm inside the inner norm. Then $H(\nu)$ can be replaced by $H(y),\left(\nu^{-1}+x\right)$ by $\left(y^{-1}+x\right)$ and $\chi_{[0, \nu]}$ 
by $\chi_{[0,2 y]}$. This produces the bound

$$
\left\|\sup _{\nu \geq 4}\right\| \frac{x^{a+\frac{\alpha}{2}}(1+x)^{b-a}}{\left(y^{-1}+x\right)^{\frac{1}{4}+\frac{\alpha}{2}}} \chi_{[0,2 y]}(x) \chi_{[\nu / 2,3 \nu / 2]}(y) H(y) f(y)\left\|_{p, y}\right\|_{p, x} .
$$

Next replace $\chi_{[\nu / 2,3 \nu / 2]}(y)$ with $\chi_{[2, \infty)}(y)$, and reverse the order of integration to get the bound $C\|f(y) L(y)\|_{p, y}$, where

$$
L(y)=\chi_{[2, \infty)}(y) H(y)\left\|\frac{x^{a+\frac{\alpha}{2}}(1+x)^{b-a}}{\left(y^{-1}+x\right)^{\frac{1}{4}+\frac{\alpha}{2}}} \chi_{[0,2 y]}(x)\right\|_{p, x} .
$$

The proof is then completed by showing that $L(y)$ is a bounded function of $y$ for $y \geq 2$. Routine computation with special attention paid to the cases $a=1 / 4-1 / p$, $b=1 / 4-1 / p$ and $p=4 / 3$ completes this part. The procedure is like that used to estimate (5.3) and its variants. The conditions used are (2.14), (2.15), (2.20), (2.21), (2.23), (2.24) and the fact that equality can not hold in both members of the following pairs: (2.14) and (2.15), (2.14) and (2.23), 2.15) and (2.24), (2.20) and (2.23), 2.23) and (2.24).

\section{PARTS 10-12: $x$ NEAR $y$}

In this section there is the added complication of having terms of the form $|x-y|$. This is also the only part in which facts about the Hardy-Littlewood maximal function are used. We will prove the following two lemmas.

Lemma (6.1). If $\alpha>-1, \delta>0$, (2.11), 2.21), $\nu \geq 4$ and $j=10$ or 12, then (2.38) holds with $C$ independent of $f, \nu$ and $x$. This is also true for $j=11$ with the additional assumption (2.28).

Lemma (6.2). If $\alpha>-1, \delta>0,1 \leq p \leq \infty$, (2.11), (2.21), $\nu \geq 4$ and $j=10$ or 12 , then (2.36) holds with $C$ independent of $f$ and $\nu$. This is also true for $j=11$ with the additional assumptions (2.20) and (2.22).

To consider the case $j=10$ observe first that

$$
\tau_{10} \leq C \frac{\nu^{1 / 2} x^{a-A+\frac{\delta}{2}}(1+x)^{b-a+A-B}}{\left[x+\nu(x-y)^{2}\right]^{\frac{1+\delta}{2}}} .
$$

Because of (2.11) and (2.21), we have $x^{a-A}(1+x)^{b-a+A-B} \leq 1$. Using this fact we get

$$
\int_{0}^{\infty} \tau_{10}|f(y)| d y \leq C \nu^{\frac{1}{2}} x^{\frac{\delta}{2}} \int_{\max (x / 2,3 / \nu-x)}^{\min (2 x, \nu-x)} \frac{|f(y)| d y}{\left[x+\nu(x-y)^{2}\right]^{\frac{1+\delta}{2}}} .
$$

By Theorem 2, page 62 of $\left[8\right.$, the integral is bounded by $C \nu^{-\frac{1}{2}} x^{-\frac{\delta}{2}} M f(x)$, where $M f$ denotes the Hardy-Littlewood maximal function of $f$. This completes the proof of Lemma (6.1) for this part. This part of Lemma (6.2) for $p>1$ follows immediately from what we have just proved and the standard norm inequality for the maximal function, Theorem 1 , page 5 of 8 . For $p=1$ integrating the expression on the right side of (6.3) and interchanging the order of integration will complete the proof. 
For $j=12$ note that at least one of $x$ and $y$ must be greater than $3 \nu / 2$ and both are greater than 2 . Therefore, since

$$
\int_{0}^{\infty} \tau_{12}|f(y)| d y \leq C \nu^{-\delta} x^{b-B+\delta} \int_{x / 2}^{2 x}\left(e^{-\xi x}+e^{-\xi y}\right)|f(y)| d y,
$$

the results follow immediately in the same way that they did for the case $j=10$.

To prove Lemma (6.1) for $j=11$ observe first that $\tau_{11}=0$ if $x$ and $y$ are not both in the interval $[\nu / 3,2 \nu]$. To prove Lemma [6.1) for this part we start with the fact that $\tau_{11}$ has the bound

$$
C \frac{\left(\nu^{1 / 3}+|x-\nu|\right)^{-1 / 4}\left(\nu^{1 / 3}+|y-\nu|\right)^{-1 / 4}\left(\nu^{\frac{1}{3}}+|x-\nu|+|y-\nu|\right)^{1+\delta}}{\nu^{B-b+\frac{\delta}{2}}\left[\nu+(x-y)^{2}\left(\nu^{1 / 3}+|x-\nu|+|y-\nu|\right)\right]^{(1+\delta) / 2}} .
$$

To prove (2.38) for $j=11$ and $|x-\nu| \leq \nu^{1 / 3}$, we will show first that for these values of $x$

$$
\left(\nu^{1 / 3}+|x-y|\right)^{3} \leq C\left[\nu+(x-y)^{2}\left(\nu^{1 / 3}+|x-\nu|+|y-\nu|\right)\right] .
$$

For $|x-y| \leq 2 \nu^{1 / 3}$ this is trivial. For $|x-y| \geq 2 \nu^{1 / 3}$ we have $|x-y| \leq|x-\nu|+|y-\nu|$, and (6.5) follows for this case also since the left side is bounded by $C\left(\nu+|x-y|^{3}\right)$. Note also that for this case we can replace $\nu^{1 / 3}+|x-\nu|$ with $\nu^{1 / 3}$ and $\nu^{1 / 3}+|y-\nu|$ with $\nu^{1 / 3}+|x-y|$. Using (6.5) to replace the denominator in (6.4), we see that the left side of (2.38) is bounded by

$$
C \int_{\nu / 3}^{2 \nu} \frac{\nu^{b-B-\frac{\delta}{2}-\frac{1}{12}}}{\left(\nu^{1 / 3}+|y-x|\right)^{\frac{3}{4}+\frac{\delta}{2}}}|f(y)| d y
$$

For $\delta \leq 1 / 6$ this is bounded by $C \nu^{b-B-\delta+1 / 6} M f(x)$ which is sufficient by (2.28). For $\delta \geq 1 / 6$ this is bounded by the value at $\delta=1 / 6$ since the value decreases as $\delta$ increases. The inequality (2.21) will then complete this part. This completes the proof of (2.38) for $|x-\nu| \leq \nu^{1 / 3}$.

For $|x-\nu| \geq \nu^{1 / 3}$ the integral in (2.38) will be split into three pieces: $|x-y| \leq$ $|x-\nu| / 2,|x-\nu| / 2 \leq|x-y| \leq 2|x-\nu|$ and $|x-y| \geq 2|x-\nu|$. In the first part $|y-\nu| \approx|x-\nu|$ and we have the estimate

$$
C \int_{|x-y| \leq|x-\nu| / 2} \frac{\nu^{b-B-\delta / 2}|x-\nu|^{\delta+1 / 2}}{\left[\nu+(x-y)^{2}|x-\nu|\right]^{(1+\delta) / 2}}|f(y)| d y .
$$

This is bounded by $C \nu^{b-B-\delta}|x-\nu|^{\delta} M f(x)$. Then since $|x-\nu| \leq \nu,(2.21)$ completes this part. For the second part we replace $\left(\nu^{\frac{1}{3}}+|y-\nu|\right)^{-1 / 4}$ by $\nu^{-1 / 12}, \nu^{1 / 3}+|x-\nu|+$ $|y-\nu|$ by $|x-\nu|$ and $(x-y)^{2}$ by $(x-\nu)^{2}$ to get $\tau_{11} \leq \nu^{b-B-\delta / 2-1 / 12}|x-\nu|^{-3 / 4-\delta / 2}$ and the integral is bounded by the quantity $C \nu^{b-B-\delta / 2-1 / 12}|x-\nu|^{1 / 4-\delta / 2} M f(x)$. For $\delta \leq 1 / 6$ the exponent of $|x-\nu|$ is positive and we can replace $|x-\nu|$ with $\nu$. This gives the estimate $\nu^{b-B-\delta+1 / 6} M f(x)$ directly for $\delta \leq 1 / 6$ and the estimate $\nu^{b-B} M f(x)$ for $\delta>1 / 6$ using the fact that the expression decreases as $\delta$ increases. Inequalities (2.28) and (2.21) complete this part. For the third part we have $|y-\nu| \approx$ $|x-y|$ and get the estimate

$$
C \nu^{b-B-\delta / 2}|x-\nu|^{-1 / 4} \int_{2|x-\nu| \leq|x-y| \leq \nu}|y-x|^{-\frac{3}{4}-\frac{\delta}{2}}|f(y)| d y .
$$


Since $|x-\nu| \geq \nu^{1 / 3}$, we can replace $|x-\nu|^{-1 / 4}$ by $\nu^{-1 / 12}$ and get the same estimate as in the last part for $\delta \leq 1 / 6$. The case $\delta>1 / 6$ is also treated as before. This completes the proof of Lemma (6.1).

To prove Lemma (6.2) for $j=11$ note first that $\tau_{11}$ for a value of $\delta$ greater than $1 / 6$ is bounded by a constant times its value at $\delta=1 / 6$ and that the hypotheses are the same for all $\delta \geq 1 / 6$. We may, therefore, in the proof assume that $0<\delta \leq 1 / 6$. The left side of (2.36) is bounded by the sum of

$$
\left\|\chi_{\left[0, \nu^{1 / 3}\right]}(|x-\nu|)\left(\int_{\nu / 3}^{2 \nu} \tau_{11}|f(y)| d y\right)\right\|_{p}
$$

and

$$
\left\|\chi_{\left[-2 \nu / 3,-\nu^{1 / 3}\right] \cup\left[\nu^{1 / 3}, 2 \nu\right]}(x-\nu)\left(\int_{\nu / 3}^{2 \nu} \tau_{11}|f(y)| d y\right)\right\|_{p} .
$$

Using the same estimate for $\tau_{11}$ as was used to obtain (6.6) and applying Hölder's inequality to the inner integral, we see that (6.8) is bounded by $\|f(y)\|_{p}$ times

$$
C \nu^{b-B-\frac{\delta}{2}-\frac{1}{12}}\left\|\chi_{\left[0, \nu^{1 / 3}\right]}(|x-\nu|)\right\| \chi_{[\nu / 3,2 \nu]}(y)\left(\nu^{1 / 3}+|y-x|\right)^{-\frac{3}{4}-\frac{\delta}{2}}\left\|_{p^{\prime}, y}\right\|_{p, x} .
$$

In the inner norm $x$ can be replaced by $\nu$ and the two norms can be evaluated to give an upper bound of

$$
C \nu^{b-B-\frac{\delta}{2}-\frac{1}{12}} \nu^{\frac{1}{3 p}}\left(\nu^{\frac{1}{4}-\frac{\delta}{2}-\frac{1}{p}}+\nu^{\frac{1}{12}-\frac{\delta}{6}-\frac{1}{3 p}}\right)
$$

if $-p^{\prime}(3+2 \delta) / 4 \neq-1$ and

$$
C \nu^{b-B-\frac{\delta}{2}-\frac{1}{12}+\frac{1}{3 p}}(\log \nu)^{1-\frac{1}{p}}
$$

if $-p^{\prime}(3+2 \delta) / 4=-1$. The conditions (2.21) and (2.22) complete the estimation of (6.10). If $-p^{\prime}(3+2 \delta) / 4=-1$, then $\delta=\frac{1}{2}-\frac{2}{p}$. Replacing $\delta$ in (6.11) by this value gives an estimate of

$$
C \nu^{b-B-\frac{1}{3}+\frac{4}{3 p}}(\log \nu)^{1-\frac{1}{p}} .
$$

Since $p=4 /(1-2 \delta)>4$, the inequality (2.21) completes this part.

To estimate (6.9) we split the inner integral as in the proof of Lemma (6.1) into parts with $|y-x| \leq|x-\nu| / 2,|x-\nu| / 2 \leq|y-x| \leq 2|x-\nu|$ and $2|x-\nu| \leq|x-y|$. In the first of these the inner integral is bounded by (6.7). As in the proof of Lemma (6.1), the condition (2.21) implies that (6.7) is bounded by $C M f(x)$ and completes this part for $p>1$. For $p=1$ change $|x-\nu|$ to $|y-\nu|$. Then estimate the $L^{1}$ norm by interchanging the norm and the integral. For parts two and three of (6.9) we use the same estimates of $\tau_{11}$ as in the proof of Lemma (6.1) except that in part two $\left(\nu^{\frac{1}{3}}+|y-\nu|\right)^{-1 / 4}$ should be retained and not replaced by $\nu^{-1 / 12}$. Then applying Hölder's inequality to the inner integral shows that these parts are bounded respectively by $\|f(y)\|_{p}$ times

$$
C\left\|\frac{\chi_{\left[\nu^{1 / 3}, \nu\right]}(|x-\nu|)}{\nu^{B-b+\frac{\delta}{2}}|x-\nu|^{\frac{3}{4}+\frac{\delta}{2}}}\right\| \chi_{|x-\nu| / 2,2 \mid x-\nu]}(|y-x|)\left(\nu^{\frac{1}{3}}+|y-\nu|\right)^{-\frac{1}{4}}\left\|_{p^{\prime}, y}\right\|_{p, x}
$$


and

$$
C\left\|\chi_{\left[\nu^{1 / 3}, \nu\right]} \frac{\nu^{b-B-\frac{\delta}{2}}}{|x-\nu|^{1 / 4}}\right\| \chi_{[2|x-\nu|, \nu]}(|y-x|)|y-x|^{-\frac{3}{4}-\frac{\delta}{2}}\left\|_{p^{\prime}, y}\right\|_{p, x} .
$$

The proof can then be completed by showing that (6.12) and (6.13) are bounded by constants independent of $\nu$.

For (6.12) computing the inner norm gives the estimate

$$
C\left\|\chi_{\left[\nu^{1 / 3}, \nu\right]}(|x-\nu|) \frac{\nu^{b-B-\frac{\delta}{2}}}{|x-\nu|^{\frac{3}{4}+\frac{\delta}{2}}}\left(\begin{array}{ll}
\nu^{1 / 4-1 /(3 p)}, & p<4 / 3 \\
(\log \nu)^{1 / 4}, & p=4 / 3 \\
|x-\nu|^{3 / 4-1 / p}, & p>4 / 3
\end{array}\right)\right\|_{p} .
$$

Then performing the $x$ integration produces the estimate

$$
C \nu^{b-B}\left(\begin{array}{lr}
\nu^{-\delta-\frac{1}{2}+\frac{2}{3 p}} & 1<p<\frac{4}{3+2 \delta} \\
\nu^{-\frac{\delta}{2}+\frac{1}{4}-\frac{1}{3 p}}(\log \nu)^{1 / p} & p=\frac{4}{3+2 \delta} \\
\nu^{-\frac{2 \delta}{3}} & \frac{4}{3+2 \delta}<p<\frac{4}{3} \\
\nu^{-\frac{2 \delta}{3}}(\log \nu)^{1 / 4} & p=\frac{4}{3} \\
\nu^{-\frac{2 \delta}{3}} & \frac{4}{3}<p
\end{array}\right) .
$$

In the first case (2.20) proves the boundedness; for the others apply (2.21).

For (6.13) computing the inner norm gives an estimate of

$$
C\left\|\chi_{\left[\nu^{1 / 3}, \nu\right]}(|x-\nu|) \frac{\nu^{b-B-\frac{\delta}{2}}}{|x-\nu|^{1 / 4}}\left(\begin{array}{ll}
|x-\nu|^{\frac{1}{4}-\frac{\delta}{2}-\frac{1}{p}} & p<\frac{4}{1-2 \delta} \\
(\log \nu)^{1-\frac{1}{p}} & p=\frac{4}{1-2 \delta} \\
\nu^{\frac{1}{4}-\frac{\delta}{2}-\frac{1}{p}} & p>\frac{4}{1-2 \delta}
\end{array}\right)\right\|_{p} .
$$

Performing the $x$ integration produces the estimate

$$
C \nu^{b-B}\left(\begin{array}{ll}
\nu^{-\frac{2 \delta}{3}} & p<\frac{4}{1-2 \delta} \\
\nu^{-\frac{2 \delta}{3}}(\log \nu)^{1-\frac{1}{p}} & p=\frac{4}{1-2 \delta} \\
\nu^{-\delta+\frac{1}{6}-\frac{2}{3 p}} & p>\frac{4}{1-2 \delta}
\end{array}\right)
$$

The condition (2.21) shows the first two are bounded, and (2.22) shows it for the third. This completes the proof of Lemma (6.2).

\section{NeCESSity RESUlts}

The obvious way to prove that the conditions in $\S 2$ are necessary for (1.1) or (1.2) would be to use an asymptotic estimate for the kernel. This, unfortunately, is not available except in a very limited way as derived in $\S 8$. Standard procedures, however, will prove that all the conditions in $N_{p}$ except (2.9), (2.12) and the pair restrictions for these inequalities are necessary for (1.1). This is done in this section. Inequalities (2.9), (2.12) and their pair restrictions will be proved necessary in $\S 9$; the proofs are based on an inequality proved in $\S 8$. This will complete the proof of the necessity part of Theorem (2.29). The $N_{p}$ conditions are, of course, necessary for (1.2). In $\S 9$ we show in addition that (2.26) is necessary for (1.2) and complete the necessity part of Theorem (2.32). 
The proofs that (2.11) and (2.21) are necessary are based on the fact that $\sigma_{n}^{(\alpha, \delta)}\left(\chi_{[r, 2 r]}, x\right)$ converges to $\chi_{[r, 2 r]}(x)$ almost everywhere as $n \rightarrow \infty$ by Theorem (2.34). Therefore, by Fatou's lemma for $p<\infty$ or trivially for $p=\infty$ we have

$$
\left\|x^{a}(1+x)^{b-a} \chi_{[r, 2 r]}(x)\right\|_{p} \leq \liminf _{n \rightarrow \infty}\left\|x^{a}(1+x)^{b-a} \sigma_{n}^{(\alpha, \delta)}\left(\chi_{[r, 2 r]}, x\right)\right\|_{p} .
$$

Applying (1.1) then produces

$$
\left\|x^{a}(1+x)^{b-a} \chi_{[r, 2 r]}(x)\right\|_{p} \leq C\left\|x^{A}(1+x)^{B-A} \chi_{[r, 2 r]}(x)\right\|_{p} .
$$

By taking $r$ large we see from this that $b-B \leq 0$, and by taking $r$ small we get $A-a \leq 0$.

The derivation of the other necessary conditions in this section is essentially standard as found, for example, in the proof of Theorem 5.1.2, page 113 of [12]. For a bounded $f$ with support a compact subset of $(0, \infty)$, the Laguerre coefficients $a_{j}=\int_{0}^{\infty} f(x) \mathcal{L}_{j}^{(\alpha)} d x$ exist and are finite. Then since

$$
\sigma_{k}^{(\alpha, \delta)}(f, x)=\left(1 / A_{k}^{\delta}\right) \sum_{j=0}^{k} A_{k-j}^{\delta} a_{j} \mathcal{L}_{j}^{(\alpha)}(x),
$$

we have

$$
a_{n} \mathcal{L}_{n}^{(\alpha)}(x)=\sum_{k=0}^{n} A_{n-k}^{-\delta-2} A_{k}^{\delta} \sigma_{k}^{(\alpha, \delta)}(f, x) .
$$

Multiplying this equality by $x^{a}(1+x)^{b-a}$, taking the $L^{p}$ norm of both sides, using Minkowski's inequality on the right and (1.1) gives the inequality

$$
\left|a_{n}\right|\left\|x^{a}(1+x)^{b-a} \mathcal{L}_{n}^{(\alpha)}(x)\right\|_{p} \leq C\left(\sum_{j=0}^{n}\left|A_{n-k}^{-\delta-2} A_{k}^{\delta}\right|\right)\left\|x^{A}(1+x)^{B-A} f(x)\right\|_{p} .
$$

Now use the estimate $\left|A_{k}^{\delta}\right| \leq C(k+1)^{\delta}$ to show that the sum is bounded by $C(n+1)^{\delta}$. This and the definition of $a_{n}$ then produces

$$
\left|\int_{0}^{\infty} f(x) \mathcal{L}_{n}^{(\alpha)}(x) d x\right|\left\|x^{a}(1+x)^{b-a} \mathcal{L}_{n}^{(\alpha)}(x)\right\|_{p} \leq C(n+1)^{\delta}\left\|x^{A}(1+x)^{B-A} f(x)\right\|_{p} .
$$

Next, if $p>1$ take

$$
f(x)=\left[x^{-A}(1+x)^{A-B}\right]^{p^{\prime}}\left|\mathcal{L}_{n}^{(\alpha)}(x)\right|^{1 /(p-1)} \operatorname{sgn}\left[\mathcal{L}_{n}^{(\alpha)}(x)\right] \chi_{[1 / m, m]}(x)
$$

where $m>0$. If $p=1$, take $f(x)=\operatorname{sgn}\left(\mathcal{L}_{n}^{(\alpha)}(x)\right) \chi_{E(m)}(x)$ where $E(m)$ is a subset of $[1 / m, m]$, has positive measure, is contained in an interval of the form $[r, 2 r]$ and such that for $x$ in $E(m)$

$$
x^{-A}(1+x)^{A-B}\left|\mathcal{L}_{n}^{(\alpha)}(x)\right| \geq \frac{1}{2} \sup _{[1 / m, m]} x^{-A}(1+x)^{A-B}\left|\mathcal{L}_{n}^{(\alpha)}(x)\right| .
$$

Dividing by the norm on the right and letting $m \rightarrow \infty$ then shows that

$$
\left\|x^{-A}(1+x)^{A-B} \mathcal{L}_{n}^{(\alpha)}(x)\right\|_{p^{\prime}}\left\|x^{a}(1+x)^{b-a} \mathcal{L}_{n}^{(\alpha)}(x)\right\|_{p} \leq C(n+1)^{\delta} .
$$

The necessity of (2.10), (2.13)-(2.20), (2.22)-(2.24) and their pair conditions then follows easily by use of the following lemma on the two norms on the left side of (7.1). 
Lemma (7.2). If $\alpha>-1, \nu=4 n+2 \alpha+2$ and $1 \leq p \leq \infty$, then

$$
\left\|x^{a}(1+x)^{b-a} \mathcal{L}_{n}^{(\alpha)}(x)\right\|_{p} \geq C\left(\nu^{-1 / 4}+\nu^{-a-1 / p}+\nu^{b-1 / 2+1 / p}+\nu^{b-1 / 3+1 /(3 p)}\right) .
$$

In addition,

$$
\left\|x^{a}(1+x)^{b-a} \mathcal{L}_{n}^{(\alpha)}(x)\right\|_{p} \geq C \nu^{-1 / 4}(\log \nu)^{1 / p}
$$

if $a=1 / 4-1 / p$ or $b=1 / 4-1 / p$,

$$
\left\|x^{a}(1+x)^{b-a} \mathcal{L}_{n}^{(\alpha)}(x)\right\|_{4} \geq C \nu^{b-1 / 4}(\log \nu)^{1 / 4},
$$

and

$$
\left\|x^{a}(1+x)^{b-a} \mathcal{L}_{n}^{(\alpha)}(x)\right\|_{p}=\infty
$$

if $a \leq-\alpha / 2-1 / p$ and $p<\infty$ or if $a<-\alpha / 2$ and $p=\infty$.

To prove Lemma (17.2) we start with the fact, (5.1.7) on page 101 of [11], that $L_{n}^{(\alpha)}(0)=\left(\begin{array}{c}n+\alpha \\ n\end{array}\right)$. By continuity, for each $n$ there is a number $h(n)>0$ such that $L_{n}^{(\alpha)}(x) \geq(1 / 2)\left(\begin{array}{c}n+\alpha \\ n\end{array}\right)$ for $0 \leq x \leq h(n)$. From this we have

$$
\mathcal{L}_{n}^{(\alpha)}(x) \geq C \nu^{\alpha / 2} x^{\alpha / 2}, \quad 0<x<h(n) .
$$

From Theorem 8.22.5, page 199 of [11] we also have

$$
\mathcal{L}_{n}^{(\alpha)}(x)=\pi^{-1 / 2}(n x)^{-1 / 4} \cos \left[2(n x)^{1 / 2}-\alpha \pi / 2-\pi / 4\right]+O\left((n x)^{-3 / 4}\right)
$$

uniformly for $1 / n \leq x \leq 1$; and from (1.5.2), page 27 of [12]

$$
\mathcal{L}_{n}^{(\alpha)}(x)=\frac{(2 / \pi)^{1 / 2}(-1)^{n}}{x^{1 / 4}(\nu-x)^{1 / 4}} \cos g+O\left((\nu x)^{-3 / 4}+\frac{\nu^{1 / 4}}{(\nu-x)^{7 / 4}}\right)
$$

uniformly for $1 \leq x \leq \nu-\nu^{1 / 3}$, where $g=[(\nu(2 \theta-\sin 2 \theta)-\pi] / 4$ and $\theta=$ $\cos ^{-1}\left(x^{1 / 2} \nu^{-1 / 2}\right)$. (For a derivation of this and the definition of $\theta$ not given in [12] see pages 453-4 of [6].) Lemma (7.2) follows by using the estimates (7.3)-(7.5).

$$
\text { 8. LOWER BOUNDS FOR } \mathcal{K}_{n}^{(\alpha, \delta)}(x, y)
$$

The result to be proved here is the following.

Lemma (8.1). If $\alpha>-1$ and $\delta>0$, then there are positive constants $C, H, L$ and $h$, independent of $x, y$ and $n$, a positive constant $N$, independent of $x$ and $y$, and $a$ set $E=E(n) \subset[1 / \nu, \nu / 2]$ satisfying

$$
|E \cap[r, 9 r]| \geq r
$$

for $H / \nu \leq r \leq L \nu / 9$, such that

$$
\mathcal{K}_{n}^{(\alpha, \delta)}(x, y) \geq C x^{-\delta / 2-3 / 4} y^{\alpha / 2} \nu^{\alpha / 2-\delta / 2+1 / 4}
$$

for $n \geq 1, x \in E \cap[H / \nu, L \nu]$ and $y \in[0, h / \nu]$, and

$$
\mathcal{K}_{n}^{(\alpha, \delta)}(x, y) \geq C \nu^{\alpha / 2-\delta-1 / 3} y^{\alpha / 2}
$$

for $n$ even, $n \geq N, x \in[\nu-8, \nu]$ and $y \in[0, h / \nu]$. 
The proof will make extensive use of the notation and proofs in [14]. Define

$$
K_{n}^{(\alpha, \delta)}(x, y)=\frac{1}{A_{n}^{\delta}} \sum_{i=0}^{n} A_{n-i}^{\delta} \frac{i !}{\Gamma(\alpha+i+1)} L_{i}^{(\alpha)}(x) L_{i}^{(\alpha)}(y)
$$

and

$$
M_{n}^{(\alpha)}(x)=(\nu / x)^{\alpha / 2} e^{x / 2} \mathcal{M}_{n}^{(\alpha)}(x)
$$

and note that

$$
K_{n}^{(\alpha, \delta)}(x, y)=e^{(x+y) / 2}(x y)^{-\alpha / 2} \mathcal{K}_{n}^{(\alpha, \delta)}(x, y)
$$

and

$$
\left|L_{n}^{(\alpha)}(x)\right| \leq C M_{n}^{(\alpha)}(x)
$$

uniformly for $x \geq 0$ and $n$ a nonnegative integer. We will first prove the following.

Lemma (8.7). If $\alpha>-1$ and $m$ is a positive integer, then there is a $C$, independent of $n, x$ and $y$, such that

$$
\left|\frac{\partial}{\partial y} K_{n}^{(\alpha, m)}(x, y)\right| \leq C \nu^{1-\alpha-m}\left(\frac{\nu+x}{\nu^{-1}+x}\right)^{(m+1) / 2} M_{n}^{(\alpha)}(x) M_{n}^{(\alpha)}(y)
$$

for $n \geq 0,0 \leq y \leq 1 / \nu$ and $2 y \leq x$.

To prove Lemma (8.7) we may assume $n \geq 1$ since the result is trivial for $n=0$. If $x \leq 2 / \nu$ use the fact that

$$
(d / d y) L_{n}^{(\alpha)}(y)=-L_{n-1}^{(\alpha+1)}(y)
$$

and (8.6) directly in the definition (8.3) to get

$$
\left|\frac{\partial}{\partial y} K_{n}^{(\alpha, m)}(x, y)\right| \leq C n^{-m} \sum_{i=0}^{n}(n-i+1)^{m}(i+1)^{-\alpha}(i+1)^{\alpha}(i+1)^{\alpha+1} .
$$

The right side is easily seen to have the bound $C \nu^{\alpha+2}$, and this is bounded by the right side of the conclusion of Lemma (8.7).

For the rest of the proof we will assume, therefore, that $n \geq 1$ and $x \geq 2 / \nu$. The proof is based on expression (35) in [14, namely

$$
\begin{aligned}
& K_{n}^{(\alpha, m)}(x, y)=\frac{(x-y)^{-1-2 m} n !}{\left(\begin{array}{c}
m+n \\
n
\end{array}\right) \Gamma(n+\alpha+1)}\left[x y(x-y) A_{m} A_{n}^{(\alpha)}(x) A_{n}^{(\alpha)}(y)\right. \\
& \left.-x B_{m} A_{n}^{(\alpha)}(x) L_{n}^{(\alpha)}(y)+y \bar{B}_{m} L_{n}^{(\alpha)}(x) A_{n}^{(\alpha)}(y)+(x-y) C_{m} L_{n}^{(\alpha)}(x) L_{n}^{(\alpha)}(y)\right],
\end{aligned}
$$

where $A_{n}^{(\alpha)}(x)=2 L_{n}^{(\alpha+1)}(x)-L_{n}^{(\alpha)}(x)$ and $A_{m}, B_{m}, \bar{B}_{m}$ and $C_{m}$ are polynomials in $x, y$ and $n$. For polynomials $p(x, y, n)$ in these variables there is a unique representation in the form $\sum a_{i, j, k}(x-y)^{i}(x+y)^{j}(2 \nu-x-y)^{k}$ and we define $p^{\#}(x, y, n)=\sum\left|a_{i, j, k} \| x-y\right|^{i}|x+y|^{j}\left(|\nu-x|+|\nu-y|+\nu^{1 / 3}\right)^{k}$. It is immediate that

$$
|p(x, y, n)| \leq p^{\#}(x, y, n)
$$

and that, for $(x, y, n)$ satisfying the conditions of the lemma and $x \geq 2 / \nu$, that

$$
\left|\frac{\partial}{\partial y} p(x, y, n)\right| \leq C x^{-1} p^{\#}(x, y, n) .
$$


Under these condition it is shown in the proof of Lemma D.2 in $\S 3$ of 14 that $A_{m}^{\#} \leq C A_{m}^{*}, B_{m}^{\#}+\bar{B}_{m}^{\#} \leq C\left(B_{(+), m}^{*}+x^{-1} B_{(-), m}^{*}\right)$ and $C_{m}^{\#} \leq C_{m}^{*}$. The quantities on the right sides of these inequalities are defined in Proposition D.1 in $\S 3$ of 14 and are shown there to have the respective bounds $C\left[x^{3}(\nu+x)\right]^{(m-1) / 2}, C\left[x^{3}(\nu+x)\right]^{m / 2}$ and $C x(\nu+x)\left[x^{3}(\nu+x)\right]^{(m-1) / 2}$ for $x, y$, and $n$ satisfying the conditions of this part of the proof. Combining these estimates with (8.10) and 8.11) shows that

$$
\begin{gathered}
\left|A_{m}\right|+x\left|\frac{\partial A_{m}}{\partial y}\right| \leq C\left[x^{3}(\nu+x)\right]^{(m-1) / 2} \\
\left|B_{m}\right|+\left|\bar{B}_{m}\right|+x\left(\left|\frac{\partial B_{m}}{\partial y}\right|+\left|\frac{\partial \bar{B}_{m}}{\partial y}\right|\right) \leq C\left[x^{3}(\nu+x)\right]^{m / 2}
\end{gathered}
$$

and

$$
\left|C_{m}\right|+x\left|\frac{\partial C_{m}}{\partial y}\right| \leq C x(\nu+x)\left[x^{3}(\nu+x)\right]^{(m-1) / 2} .
$$

By Proposition C.1 in $\S 3$ of [14] for $x \geq 2 / \nu$ we have

$$
\left|A_{n}^{(\alpha)}(x)\right| \leq C\left(\frac{\nu^{1 / 3}+|x-\nu|}{x}\right)^{1 / 2} M_{n}^{(\alpha)}(x),
$$

while from (8.8), (8.6) and the definition of $A_{n}^{(\alpha)}$ we have

$$
\left|L_{n}^{(\alpha)}(y)\right|+\nu^{-1}\left|\frac{\partial L_{n}^{(\alpha)}(y)}{\partial y}\right| \leq C M_{n}^{(\alpha)}(y)
$$

and

$$
\left|A_{n}^{(\alpha)}(y)\right|+\nu^{-1}\left|\frac{\partial A_{n}^{(\alpha)}(y)}{\partial y}\right| \leq C \nu M_{n}^{(\alpha)}(y) .
$$

The proof of Lemma 8.7 is then completed by differentiating the right side of (8.9) with respect to y and using (8.6) and (8.12) - (8.17) to estimate the absolute value of each resulting term.

Next we need an estimate of $\left|(\partial / \partial y) K_{n}^{(\alpha, \delta)}(x, y)\right|$, valid for all $\delta>0$; this is given by Lemma (8.18).

Lemma (8.18). If $\alpha>-1$ and $\delta>0$, then there is a $C$, independent of $x, y$ and $n$, such that

$$
\left|\frac{\partial}{\partial y} K_{n}^{(\alpha, \delta)}(x, y)\right| \leq C \frac{e^{x / 2} \nu^{\alpha / 2-\delta / 2+3 / 2} x^{-\alpha / 2-\delta / 2-3 / 4}}{\left(\nu^{1 / 3}+|x-\nu|\right)^{1 / 4}}
$$

for $n \geq 0,0 \leq y \leq 1 / \nu$ and $2 / \nu \leq x \leq \nu$.

This is proved by differentiating the conclusion of Proposition A.1 in $\S 5$ of [14] and using the estimate of Lemma (8.7) with $\vartheta=\left\lfloor n-(\nu / x)^{1 / 2}\right\rfloor$. The proof is like the proof of Theorem B.1 in $\S 5$ of [14]. For the range of $x$ and $y$ of this lemma the estimates for $\left|(\partial / \partial y) K_{n}^{(\alpha, j)}(x, y)\right|$ given by Lemma (8.7) are bounded by $\nu$ times the estimates used for $\left|K_{n}^{(\alpha, j)}(x, y)\right|$ in the proof of Theorem B.1 in $\S 5$ of [14]. Therefore, that proof gives a bound here of $\nu$ times the bound obtained there and completes the proof of this lemma. 
To complete the proof of the first part of Lemma (8.1), observe from the fact, (5.1.6) on page 101 of [11],

$$
L_{n}^{(\alpha)}(x)=\sum_{j=0}^{n}\left(\begin{array}{c}
n+\alpha \\
n-j
\end{array}\right) \frac{(-x)^{j}}{j !}
$$

used in (8.3) that

$$
K_{n}^{(\alpha, \delta)}(x, 0)=\frac{1}{A_{n}^{\delta} \Gamma(\alpha+1)} L_{n}^{(\alpha+\delta+1)}(x) .
$$

Now define the set $E$ to be the set where the cosine terms in (7.4) and (7.5) for $\mathcal{L}_{n}^{(\alpha+\delta+1)}(x)$ are greater than $1 / 2$. From the form of the arguments of the cosines the inequality (8.2) follows for $G / \nu \leq r \leq L \nu$ for suitably chosen $G \geq 2$ and $L \leq 1 / 2$. From (8.20), (2.1), (7.4) and (7.5) we have

$$
K_{n}^{(\alpha, \delta)}(x, 0) \geq C_{1} e^{x / 2} \nu^{\alpha / 2-\delta / 2+1 / 4} x^{-\alpha / 2-\delta / 2-3 / 4}\left(1-C_{2}(\nu x)^{-1 / 2}\right)
$$

for $x \in E \cap[2 / \nu, \nu / 2]$. Now with $H=\max \left(4 C_{2}^{2}, G\right)$ we get from (8.21) that

$$
K_{n}^{(\alpha, \delta)}(x, 0) \geq \frac{C_{1}}{2} e^{x / 2} \nu^{\alpha / 2-\delta / 2+1 / 4} x^{-\alpha / 2-\delta / 2-3 / 4}
$$

for $x \in E \cap[H / \nu, L \nu]$. From Lemma (8.18)

$$
\left|\frac{\partial}{\partial y} K_{n}^{(\alpha, \delta)}(x, y)\right| \leq C_{3} e^{x / 2} \nu^{\alpha / 2-\delta / 2+5 / 4} x^{-\alpha / 2-\delta / 2-3 / 4}
$$

for $0 \leq y \leq 1 / \nu$. Therefore, if $h=\min \left(1, C_{1} /\left(4 C_{3}\right)\right)$,

$$
\left|K_{n}^{(\alpha, \delta)}(x, y)-K_{n}^{(\alpha, \delta)}(x, 0)\right| \leq\left(C_{1} / 4\right) y e^{x / 2} \nu^{\alpha / 2-\delta / 2+5 / 4} x^{-\alpha / 2-\delta / 2-3 / 4}
$$

for $0 \leq y \leq h / \nu$. Then we get from (8.22) and (8.23) that

$$
K_{n}^{(\alpha, \delta)}(x, y) \geq \frac{C_{1}}{4} e^{x / 2} \nu^{\alpha / 2-\delta / 2+1 / 4} x^{-\alpha / 2-\delta / 2-3 / 4}
$$

for $y \in[0, h / \nu]$ and $x \in E \cap[H / \nu, L \nu]$. This is equivalent to the first conclusion of Lemma (8.1).

For the second conclusion we start by using (8.20) and (8.22.11), page 201 of 11] to obtain

$$
K_{n}^{(\alpha, \delta)}(x, 0) \geq C_{1} x^{-\delta-1 / 3} e^{x / 2}\left(1-C_{2} n^{-2 / 3}\right)
$$

for $n$ even and $\nu-8 \leq x \leq \nu$. From Lemma (8.18)

$$
\left|\frac{\partial}{\partial y} K_{n}^{(\alpha, \delta)}(x, y)\right| \leq C_{3} e^{x / 2} x^{2 / 3-\delta}
$$

for $0 \leq y \leq 1 / \nu$ and $\nu-8 \leq x \leq \nu$. Therefore, if $h=\min \left(1, C_{1} /\left(4 C_{3}\right)\right), 0 \leq y \leq h / \nu$ and $\nu-8 \leq x \leq \nu$, we have

$$
\left|K_{n}^{(\alpha, \delta)}(x, y)-K_{n}^{(\alpha, \delta)}(x, 0)\right| \leq \frac{C_{1}}{4} e^{x / 2} x^{-\delta-1 / 3} .
$$

Then, with $N>\left(2 C_{2}\right)^{3 / 2}$ we have

$$
K_{n}^{(\alpha, \delta)}(x, y) \geq \frac{C_{1}}{4} x^{-\delta-1 / 3} e^{x / 2}
$$

for $n \geq N, n$ even, $0<y \leq h / \nu$ and $\nu-8 \leq x \leq \nu$. The result follows from (88.5). 


\section{Conclusion of NeCESSity PROOFS}

Here we complete the proof of the necessity part of Theorem (2.29) by showing that (1.1) implies (2.9), (2.12) and the pair restrictions involving them. After that we prove as Theorem (9.3) the fact that (2.26) is necessary for (1.2). Finally we will complete the proof of Theorem (2.32) by showing that the conditions in $S_{p}$ that have not previously been shown to be necessary for (1.2) are necessary for (2.33).

To show that (2.12) is a necessary condition for (1.1), take $f(x)=\chi_{[h /(2 \nu), h / \nu]}(x)$, where $h$ is the constant in Lemma 8.1). By that lemma then

$$
\left|\sigma_{n}^{(\alpha, \delta)}(f, x)\right| \geq C \nu^{-\delta / 2-3 / 4} x^{-\delta / 2-3 / 4}
$$

for $x$ in $E \cap[H / \nu, L \nu]$. Using this in (1.1) shows that

$$
\nu^{-\delta / 2-3 / 4}\left\|\chi_{E \cap[H / \nu, L \nu]} x^{a-\delta / 2-3 / 4}(1+x)^{b-a}\right\|_{p} \leq C \nu^{-A-1 / p} .
$$

Restricting the integration in the norm to $E \cap[1,9]$ and using (8.2) then gives $\nu^{-\delta / 2-3 / 4} \leq C \nu^{-A-1 / p}$, and this implies 2.12).

If (2.11) and (2.12) were both equalities, then $a=A=3 / 4+\delta / 2-1 / p$ and (9.1) with $x$ integration restricted to $E \cap[h / \nu, 1]$ would imply

$$
\nu^{-\delta / 2-3 / 4}\left\|\chi_{E \cap[H / \nu, 1]} x^{-1 / p}\right\|_{p} \leq C \nu^{-\delta / 2-3 / 4}
$$

Since the norm in this case is bounded below by $C(\log \nu)^{1 / p}$, we have a contradiction and conclude that (2.11) and (2.12) can not both be equalities if $p<\infty$. Similarly, if (2.12) and (2.16) were both equalities we could take $f(x)=\chi_{E \cap[1, L \nu]}$ and have the same contradiction for $p<\infty$. If $p=\infty$, then (2.17) implies that (2.16) is strict. that

For (2.9) we start with the fact (from Lemma (8.1) and $\mathcal{K}_{n}^{(\alpha, \delta)}(x, y)=\mathcal{K}_{n}^{(\alpha, \delta)}(y, x)$ )

$$
\mid \mathcal{K}_{n}^{(\alpha, \delta)}\left(x, y \mid \geq C x^{\alpha / 2} y^{-\delta / 2-3 / 4} \nu^{\alpha / 2-\delta / 2+1 / 4}\right.
$$

for $x \in[0, h / \nu]$ and $y \in E \cap[H / \nu, L \nu]$. Using this in (1.1) and restricting the integration in the norm to $[h /(2 \nu), h / \nu]$ shows that

$$
\nu^{-a-\delta / 2-1 / p+1 / 4}\left|\int_{E \cap[H / \nu, L \nu]} \frac{|f(y)| d y}{y^{\delta / 2+3 / 4}}\right| \leq C\left\|\frac{f(x) \chi_{E \cap[H / \nu, L \nu]}(x)}{x^{-A}(1+x)^{A-B}}\right\|_{p} .
$$

Taking $f(x)=\chi_{[1,9]}$ in (9.2) will then show that (2.9) is necessary. If (2.9) and (2.11) were both equalities, we would have $a=A=1 / 4-\delta / 2-1 / p$. Taking $f(x)=x^{\delta / 2-1 / 4} \chi_{[H / \nu, 1]}$ in (9.2) leads to $\log \nu \leq C(\log \nu)^{1 / p}$ and shows those two equalities are incompatible if $p>1$. If (2.9) and (2.15) were both equalities, taking $f(x)=x^{\delta / 2-1 / 4} \chi_{E \cap[1, L \nu]}$ leads to the same contradiction for $p>1$ For $p=1$ (2.15) can not be an equality because of (2.14). This completes the proof of Theorem (2.29).

Theorem (9.3). If $1<p \leq \infty, \alpha>-1, \delta>0$ and (1.2) holds with $C$ independent of $f$, then (2.26) is satisfied.

To prove this, let $N, C$ and $h$ be the constants in Lemma (8.1) and let $n$ be an even integer greater than $N$ and 2. Let $f(y)=\chi_{[h /(6 \nu), h /(3 \nu)]}(y)$. From (1.2) then

$$
\left\|\chi_{[\nu, 2 \nu]}(x) x^{b} \int_{h /(6 \nu)}^{h /(3 \nu)} \mathcal{K}_{j}^{(\alpha, \delta)}(x, y) d y\right\|_{p} \leq C\left\|\chi_{[h /(6 \nu), h /(3 \nu)]} y^{A}\right\|_{p},
$$


where $j=2\lfloor(x-2 \alpha-2) / 8\rfloor+2$ and $\lfloor w\rfloor$ denotes the greatest integer less than or equal to $w$. For this choice of $j$ and $x \in[\nu, 2 \nu]$ we have $\nu(j)-8 \leq x<\nu(j)$ and $\nu(j) \leq 2 \nu+8 \leq 3 \nu$, where $\nu(j)=4 j+2 \alpha+2$. Therefore, in (9.4) $y \leq h / \nu(j)$ and by using Lemma (8.1) in (9.4) we get

$$
\left\|\chi_{[\nu, 2 \nu]}(x) x^{b+\alpha / 2-\delta-1 / 3} \int_{h /(6 \nu)}^{h /(3 \nu)} y^{\alpha / 2} d y\right\|_{p} \leq C \nu^{-A-1 / p} .
$$

Computing the norm and using the fact that the resulting inequality must hold for arbitrarily large $\nu$ then proves (2.26).

Finally, to complete the proof of Theorem (2.32) note that the only inequalities that have not already been shown to be necessary for (1.2) are (2.25), (2.27) and (2.28). We will first prove the necessity of these inequalities for (2.33) and then show that the additional pair conditions are satisfied.

Replacing $\tau$ by $\tau_{8}$ in (2.33) and taking $\nu=x$, we get the inequality

$$
\left\|\chi_{[4, \infty)}(x) x^{b-\frac{7}{12}-\delta} \int_{0}^{x / 2} \frac{y^{\frac{\alpha}{2}-A}(1+y)^{A-B}}{\left(y+x^{-1}\right)^{\frac{1}{4}+\frac{\alpha}{2}}}|f(y)| d y\right\|_{p} \leq C\|f\|_{p} .
$$

Given $w \geq 2$, let $f$ be the characteristic function of $[w / 4, w / 2]$, take the norm in (9.5) over $[w, 2 w]$ and the inner integral over $[w / 4, w / 2]$. This shows that

$$
w^{b-7 / 12-\delta+1 / p} w^{3 / 4-B} \leq C w^{1 / p} .
$$

Since this is true for all $w>2$, it follows that (2.28) holds. With $f$ the characteristic function of $[1 / 2,1]$ we see from (9.5) that

$$
\left\|\chi_{[4, \infty)}(x) x^{b-\frac{7}{12}-\delta}\right\|_{p} \leq C .
$$

from which (2.27) follows.

Next, use $\tau_{5}$ in (2.33) and take $\nu=x^{-1}$ to get

$$
\left\|\chi_{[0,1 / 4]}(x) x^{a-\frac{1}{4}+\frac{\delta}{2}} \int_{2 x}^{1} y^{-A-\frac{3}{4}-\frac{\delta}{2}}|f(y)| d y\right\|_{p} \leq C\|f\|_{p} .
$$

With $f$ the characteristic function of $[1 / 2,1]$ this gives $\left\|\chi_{[0,1 / 4]}(x) x^{a-\frac{1}{4}+\frac{\delta}{2}}\right\|_{p} \leq C$, which implies (2.25).

Of the pair conditions in $S_{p}$, one is stated for $p=1$ only and for two others, the pair (2.11) and (2.13) and the pair (2.13) and (2.26), equality in both could only occur for $p=1$. Since Theorem (2.32) only concerns $p>1$, these need not be considered. Five pair conditions are stated in the same way in the $S_{p}$ conditions and in the $N_{p}$ conditions and were proved necessary in $\S 7$. Two involve (2.25); since equality in (2.25) implies equality in (2.9), these have been treated in this section. Equality in both (2.26) and (2.27) can occur only if $p=\infty, b=\delta+7 / 12$ and $A=3 / 4$. Using these values, taking $f(x) \equiv 1$ in (9.5) and restricting the inner integral to $[1 / x, 1]$ leads to the contradiction $\left\|\chi_{[4, \infty]}(x) \int_{1 / x}^{1} y^{-1} d y\right\|_{\infty} \leq C$. Similarly, if (2.27) and (2.28) are both equalities, then $p=\infty, b=\delta+7 / 12$ and $B=3 / 4$. Taking $f(x) \equiv 1$ in (9.5) and restricting the inner integral to $[1, x / 2]$ again leads to a contradiction. This completes the proof of Theorem (2.32). 


\section{Extension of Theorem (2.30)}

The condition (2.28) is not a necessary condition for Theorem (2.30). This can be seen by starting with the fact implied by Theorem (2.30) that

$$
\left\|\sup _{n \geq 0}\left(\left|\sigma_{n}^{(\alpha, \delta)}(f, x)\right|\right)\right\|_{2} \leq C\|f(x)\|_{2}
$$

for $\delta \geq 1 / 6$. A result of Bonami and Clerc, Lemma (3.5), page 238 of [2], asserts that if the inequality (10.1) holds for one $\delta>0$, then it holds for all $\delta>0$. Therefore, (10.1) is also valid for $0<\delta<1 / 6$. This fact can be used to prove the following extension of Theorem (2.30).

Theorem (10.2). If $0<\delta<1 / 6, \alpha>-1,2 /(1+6 \delta)<p<2 /(1-6 \delta), h=1 / 2-$ $1 / p, a>\max (-3 \delta(\alpha+1)+h,-2 \delta+h), A-a \leq 0, A<\min (3 \delta(\alpha+1)+h, 2 \delta+h$,$) ,$ $a+B>\max (-5 \delta+4 h / 3,-4 \delta+2 h), A+b<3 \delta+2 h, b<3 \delta / 2+h, b-B \leq 0$ and $B>\max (-7 \delta / 2+h / 3,-5 \delta / 2+h)$, then (1.2) holds with $C$ independent of $f$.

The idea for this theorem and the proof are from the remark above Proposition 4.3 , page 325 of [10]. The method of Chapter VII, $\S 5$ of [9] is used to interpolate between inequalities of the form

$$
\int_{0}^{\infty} \frac{\left[\frac{x^{a d}}{(1+x)^{a d-b d}} \sup _{n \geq 0}\left|\sigma_{n}^{(\alpha, \delta)}(f, x)\right|\right]^{q}}{x^{2 a d}(1+x)^{2 b d-2 a d}} d x \leq C \int_{0}^{\infty} \frac{\left|x^{A d}(1+x)^{(B-A) d} f(x)\right|^{q}}{x^{2 A d}(1+x)^{2 B d-2 A d}} d x
$$

where $d=p /(p-2)$. For one end of the interpolation take $q=2$ and $\delta=s$ for suitably small positive $s$. At the other end use $\delta=1 / 6$ and $q=p_{0}$ where $1 / p_{0}=$ $1 / 2-\Delta /(2 d)$ and $\Delta=(1-6 s) /(6 \delta-6 s)$. The $q=2$ inequality is automatically satisfied because of (10.1). For $p_{0}$ we will use Theorem (2.30). Therefore, we require that the $S_{p}$ conditions hold with $p$ replaced by $p_{0}, \delta$ by $1 / 6, a$ by $a \Delta, b$ by $b \Delta$, $A$ by $A \Delta$ and $B$ by $B \Delta$. To make these inequalities true for some $s>0$ requires exactly the hypotheses of this theorem.

\section{REFERENCES}

[1] Richard Askey and Stephen Wainger, Mean convergence of expansions in Laguerre and Hermite series, Amer. J. Math. 87 (1965), 695-708. MR 32:316

[2] Aline Bonami and Jean-Louis Clerc, Sommes de Cesàro et multiplicateurs des développements en harmoniques sphériques, Trans. Amer. Math. Soc. 183 (1973), 223-263. MR 49:3461

[3] Clemens Markett, Norm estimates for Cesàro means of Laguerre expansions, approximatioins and function spaces, in Approximation and Function Spaces, North Holland, Amsterdam-New York (1981), 419-435. MR 83f:42018

[4] _ Mean Cesàro summability of Laguerre expansions and norm estimates with shifted parameter, Analysis Math. 8 (1982), 19-37. MR 83j:40004

[5] B. Muckenhoupt, Asymptotic forms for Laguerre polynomials, Proc. Amer. Math. Soc. 24 (1970), 288-292. MR 40:4503

[6] Mean convergence of Hermite and Laguerre series II, Trans. Amer. Math. Soc. 147 (1970), 433-460. MR 41:711

[7] Eileen Poiani, Mean Cesàro summability of Laguerre and Hermite series, Trans. Amer. Math. Soc. 173 (1972), 1-31. MR 46:9635

[8] Elias Stein, Singular Integrals and Differentiability Properties of Functions, Princeton University Press (1970). MR 44:7280

[9] Elias Stein and Guido Weiss, Introduction to Fourier Analysis on Euclidean Spaces, Princeton University Press (1971). MR 46:4102]

[10] Krzysztof Stempak, Almost everywhere summability of Laguerre series II, Stud. Math. 103 (3) (1992), 317-327. MR 92a:42053 
[11] Gabor Szegö, Orthogonal Polynomials, fourth ed., Amer. Math. Soc. Colloq. Publ., vol. 23, Amer. Math. Soc., Providence, R.I., 1975. MR 51:8724

[12] Sundaram Thangavelu, Lectures on Hermite and Laguerre Expansions, Princeton Univ. Press (1993). MR 94i:42001

[13] _ Summability of Laguerre expansions, Analysis Math. 16 (1990), 303-315. MR 92h:33021

[14] David Webb, Pointwise estimates of the moduli of Cesàro-Laguerre kernels, to appear.

Department of Mathematics, Rutgers University, Piscataway, New Jersey 08854-8019

E-mail address: muckenho@math.rutgers.edu

Department of Mathematics, DePaul University, Chicago, Illinois 60614-3504

E-mail address: dwebb@condor.depaul.edu 